\title{
Shifting Trust: Examining How Trust and Distrust Emerge, Transform, and Collapse in COVID-19 Information Seeking
}

\author{
Yixuan Zhang \\ Georgia Institute of Technology \\ Atlanta, GA, USA \\ yixuan@gatech.edu \\ Joseph D Gaggiano \\ Georgia Institute of Technology \\ Atlanta, GA, USA \\ jgaggiano@gatech.edu \\ Yifan Sun \\ William \& Mary \\ Williamsburg, VA, USA \\ ysun25@wm.edu
}

\author{
Nurul M Suhaimi \\ Northeastern University \\ Boston, MA, USA \\ suhaimi.n@northeastern.edu \\ Miso Kim \\ Northeastern University \\ Boston, MA, USA \\ m.kim@northeastern.edu \\ Stacy Marsella \\ Northeastern University \\ Boston, MA, USA \\ s.marsella@northeastern.edu \\ Andrea G Parker \\ Georgia Institute of Technology \\ Atlanta, GA, USA \\ andrea@cc.gatech.edu
}

\author{
Nutchanon Yongsatianchot \\ Northeastern University \\ Boston, MA, USA \\ yongsatianchot.n@northeastern.edu \\ Shivani A Patel \\ Emory University \\ Atlanta, GA, USA \\ s.a.patel@emory.edu \\ Jacqueline Griffin \\ Northeastern University \\ Boston, MA, USA \\ ja.griffin@northeastern.edu
}

\begin{abstract}
During crises like COVID-19, individuals are inundated with conflicting and time-sensitive information that drives a need for rapid assessment of the trustworthiness and reliability of information sources and platforms. This parallels evolutions in information infrastructures, ranging from social media to government data platforms. Distinct from current literature, which presumes a static relationship between the presence or absence of trust and people's behaviors, our mixed-methods research focuses on situated trust, or trust that is shaped by people's information-seeking and assessment practices through emerging information platforms (e.g., social media, crowdsourced systems, COVID data platforms). Our findings characterize the shifts in trustee (what/who people trust) from information on social media to the social media platform(s), how distrust manifests skepticism in issues of data discrepancy, the insufficient presentation of uncertainty, and how this trust and distrust shift over time. We highlight the deep challenges in existing information infrastructures that influence trust and distrust formation.
\end{abstract}

\section{CCS CONCEPTS}

- Human-centered computing $\rightarrow$ Human computer interaction (HCI); Empirical studies in HCI.

\section{KEYWORDS}

crisis informatics, information behaviors, trust, distrust, misinformation, information infrastructure, social media, mixed methods, longitudinal research, COVID-19

\section{ACM Reference Format:}

Yixuan Zhang, Nurul M Suhaimi, Nutchanon Yongsatianchot, Joseph D Gaggiano, Miso Kim, Shivani A Patel, Yifan Sun, Stacy Marsella, Jacqueline Griffin, and Andrea G Parker. 2022. Shifting Trust: Examining How Trust and Distrust Emerge, Transform, and Collapse in COVID-19 Information Seeking. In CHI Conference on Human Factors in Computing Systems (CHI '22), April 29-May 5, 2022, New Orleans, LA, USA. ACM, New York, NY, USA, 21 pages. https://doi.org/10.1145/3491102.3501889

\section{INTRODUCTION}

Due to COVID-19, 2020 and 2021 have been marked by a sense of confusion and uncertainty as people are required to navigate this new and emerging crisis. Scientific communities, governments, organizations, and individuals have all been learning and adapting to changes in the crisis as it has unfolded $[52,79]$. Consequently, COVID-19-associated regulations and guidance, as well as projections and forecasts, have also shifted frequently. The conflicting and, often, time-sensitive information created by varied sources and disseminated on diverse platforms has engendered a feeling of uncertainty. This uncertainty makes it difficult for people to determine which information sources are trustworthy and which platforms (e.g., social media, news outlets) they should rely on.
Permission to make digital or hard copies of all or part of this work for personal or
classroom use is granted without fee provided that copies are not made or distributed for profit or commercial advantage and that copies bear this notice and the full citation on the first page. Copyrights for components of this work owned by others than ACM must be honored. Abstracting with credit is permitted. To copy otherwise, or republish, to post on servers or to redistribute to lists, requires prior specific permission and/or

fee. Request permissions from permissions@acm.org.

CHI '22, April 29-May 5, 2022, New Orleans, LA, USA

(c) 2022 Association for Computing Machinery.

ACM ISBN 978-1-4503-9157-3/22/04 . \$15.00

https://doi.org/10.1145/3491102.3501889 
Meanwhile, a variety of information and communication technologies (ICTs) [37] and sociotechnical systems (STSs) have been created, adapted, and utilized to communicate crisis and risk information amidst the COVID-19 pandemic. (We define STSs as interactions between people, organizations, institutions, and ICTs [44]). For example, well-established social media platforms have been heavily utilized by the general public to access COVID-19 information [61]. Additionally, an increasing number of information platforms have been created from the ground up (e.g., crowdsourced systems, such as the COVID Tracking Project [80]) or adapted from existing platforms (e.g., data trackers from news outlets [17, 81] and public health authorities [14]) to report COVID-19 data (e.g., number of cases/deaths). The COVID-19 data provided by these platforms have served as the backbone for crisis and risk information dissemination during the pandemic [51] and have been consumed by millions of people daily [5]. Given their widespread use and the central role that they play in informing the public, these platforms need to be asked one key question: to what extent do people trust the information that they provide?

Amidst the COVID-19 pandemic, a growing body of work has demonstrated the importance of investigating this question [39]. While a majority of trust literature amidst COVID-19 has typically presumed a static, or unchanging, relationship between the presence or absence of trust and peoples' information behaviors [56], little is known about the ways in which information trust changes and evolves during a crisis. Yet, information appraisals are necessarily dynamic, particularly amidst a rapidly evolving scenario like the COVID-19 pandemic. Indeed, recent work has discussed how the general public has to constantly reassess the threat of COVID-19 to public health, reexamine the efficacy of the governmental responses to the pandemic, and reevaluate their trust in the government $[20,32]$. However, while this work has yielded insight into the political implications of evolving information appraisals during crises, two important research gaps exist. First, this work has rarely examined the role that ICTs and STSs have played in shaping the public's trust of information. Second, little work has investigated the sociotechnical context of information trust evolution amongst traditionally-marginalized populations, such as low-socioeconomic (low-SES) communities. Yet, prior research has thoroughly established the disproportionate impact that COVID19 has had on communities such as Black, Hispanic, and low-SES groups in the United States [66]. To mitigate this risk, it is crucial to ensure that these groups have access to crisis and risk information from credible resources. These disparities bring into sharp relief the importance of investigating information trust among traditionally-marginalized populations [69], given the important role that information can play in mitigating the morbidity and mortality rates in these populations during a public health crisis [86]. Furthermore, marginalized groups may have distinctive needs and perspectives regarding the use of, information from, and trust in a particular source [77, 93].

To address these research gaps, we conducted a mixed method study, which included a longitudinal survey and semi-structured interviews, to examine information practices and trust amidst the COVID-19 pandemic. Our survey results show that while COVID19 information consumption on more traditional media platforms (e.g., radio, television, and newspapers) stayed consistent over time,
COVID-19 information consumption via the internet significantly decreased over time. This decline was especially pronounced on social media. Triangulated with our interview study results, we found that our participants stopped using social media for COVID19 information consumption mainly for two reasons: 1) their fear of being potentially impacted by the mixture of true information and misinformation, and 2) their frustration about their inability to change their social media contacts' misbeliefs and mistrust. Moreover, our study suggests that there was an association between participants' distrust and their information exposure, with lower COVID-19 information exposure levels being associated with higher levels of information distrust. Additionally, our findings reveal how distrust arose from participants' skepticism of the information production processes, as well as their desire for clearer communication of data uncertainty, and how this distrust changed over time.

Overall, this paper contributes a situated perspective in examining how trust and distrust are shaped by existing information production, representation, and dissemination processes using ICTs and STSs, such as government websites, public media, crowdsourced systems, and social media, during COVID-19, as well as how this trust and distrust shift over time. Our work sheds light on the deep challenges in existing information infrastructures that have shaped the formation of public trust and distrust. We also provide implications for future work that guide the collaborative effort among stakeholders to improve the information infrastructure, to foster public trust, and to better prepare for future crises.

\section{BACKGROUND AND RELATED WORK}

Trust is critical for the formation of cohesive relationships between people, organizations, and information and the technology in which relationships are relied upon [24]. We define trust as a person's (the "truster") belief in and the willingness to rely on the trustee (i.e., a trusted party like an individual, an organization, information, or an information source in the digital age $)^{1}$. Trust in a particular information source can influence the type of information people receive, which may influence their subsequent decisions and behaviors [25]. A few related concepts include distrust, defined as the extent to which the truster believes that the trustee will work against, or at least not in the best interest of, the truster [53], and mistrust, defined as misplaced trust whereby a trustee "defaults" or betrays a person's trust [53]. In a situation of mistrust, a person finds that while they had at one point trusted the trustee, that trustee later proves to in fact not be worthy of trust. As many trust issues surrounding COVID-19 information have arisen during the pandemic $[9,49]$, it is critical to examine how people form their trust, distrust, and mistrust and the way in which these beliefs evolve over time.

\subsection{Factors Influencing Trust}

Much of the work in the trust literature specifically examines the factors that determine trust by identifying the characteristics and qualities that make up both a trustee $[15,54,73]$ and a truster [8]. On one hand, the most cited characteristics of a trustee $[15,54,73]$

\footnotetext{
${ }^{1}$ No consensus currently exists among trust scholars on which definition of trust is the most appropriate. Leveraging the literature on trust, our definition most closely aligns with McKnight et al. [55], Mayer et al. [54], and Currall et al. [18]. See Burke et al.'s review for more definitions of trust [10].
} 
include ability (i.e., the skills, competencies, and characteristics that allow a party to be influential in a specific domain), benevolence (i.e., the extent to which a trustee is believed to work in the trustor's interests), integrity (i.e., the beliefs that a trustee adheres to principles that the trustor finds acceptable), and reliability (i.e., the extent to which a source is reliable). Most work suggests that the source of crisis and risk information is the most important factor influencing a person's trust in that information source [23, 65].

Given the abundance of information available online, researchers have increasingly studied how people develop their trust in this information. This body of work has surfaced a number of factors that impact trust formation in the context of social media information. For example, on social media, people evaluate the trustworthiness of posts mostly based on the source and content [65]. There is also a body of work investigating the impacts of information credibility indicators (e.g., the presence of fact-checking or if the source is a major news outlet) in social media contexts. While such indicators can help decrease social media users' propensity to share fake news online [99], prior work argues that instead of evaluating the trustworthiness of information based on its credibility, people form their opinions and judge the veracity of information primarily based on their familiarity with its source [90]. Nonetheless, even in the context of unfamiliar accounts on social media, most users can effectively assess information authenticity and credibility, and thus the presence or absence of an authenticity indicator does not significantly influence people's willingness to share information [90]. In fact, in most cases, people do not investigate low-credibility social media posts to see how the claims were generated or why the account posted it [29].

In addition to credibility, other research has highlighted the important role that social media users have as distributors of information. Specifically, the person or account that circulates an article on social media may have a greater impact on what people think of the story than the original information producer who wrote the story [76]. As such, the credibility of the information sharers influences people's opinions about the source [76]. This variation of information assessment practices is further complicated with the high uncertainty associated with crises. Prior work has found that crisis situations are associated with a pervasive distrust in "formal" sources (e.g., mainstream news media), driving people to seek information via social media and contributing to the creation and spread of conspiracy beliefs [43].

To help people make more informed trust assessments online, an increasing body of literature has explored to use algorithmiccentered approaches to detect and improve the credibility of online information [3, 38, 70, 97]. Work in HCI has begun focusing on the enhancement of information literacy and the facilitation of trusters' information sensemaking [27]. Our work extends this body of literature in understanding the dynamics of trust-how people form their trust and distrust during the processes of information seeking in times of crisis.

\subsection{A Situated Perspective on Trust}

However, a fundamental debate within the trust literature is not only what determines trust but also whether trust is rational or affective. Some trust scholars suggest that trust is an affective construct that can vary depending on the context and experiences of a person rather than simply being a rational or an objective reality [54]. This body of work argues that trust should be conceptualized and studied as emergent states [10], or cognitive, motivational, or affective states that are dynamic and vary as a function of contextual factors, as well as inputs, processes, and outcomes. Following this perspective, trust is developed or collapsed given specific interactions and contexts [16]. This view has resonance with the similar notion of situated trust $[11,36,64]$, which emphasizes the impact that contextual factors have on the situation (e.g., trust is placed upon a specific person for a specific task within a particular situation). Understanding trust from a situated perspective enables trust scholars to understand the dynamics of trust and acquire a better understanding of how trust changes over time. Our work seeks to examine the evolution of trust and distrust formation amidst the COVID-19 pandemic.

\subsection{Trust \& COVID-19}

A growing body of work has explored two broad realms regarding trust during the COVID-19 pandemic, including the influences that the presence or absence of trust have on people's behaviors (e.g., behavioral compliance, mortality, risk perception), and the impact of the pandemic on trust [20]. Recent work by Melki et al. [56] shows that those who trust COVID-19 news from social media, interpersonal communication, and/or clerics are more likely to believe in COVID-19 misinformation than those who trust in government information sources. Additionally, prior work has suggested that misbeliefs are more common among those who are in traditionally-marginalized populations (e.g., lower levels of education and income) [30, 67, 91, 92].

However, to the best of our knowledge, there has been a lack of research that examines situated trust during COVID-19-how trust and distrust are shaped by the emerging information platforms, and how this trust and distrust change over the course of the crisis. Specifically, we refer to the situated trust regarding the emerging information platforms that have been built from the ground up in response to the COVID-19 pandemic-platforms that report and present COVID-19 data such as online dashboards-as well as the well-established platforms available prior to the pandemic, such as social media platforms. The emerging information systems, in particular, were initiated and rapidly built during the early stage of COVID-19 with the goal of providing accurate and timely COVID-19 data (e.g., number of cases/deaths) [80]. Others have been adapted from existing infrastructure by adding new components to the existing platforms, such as the COVID-19 data trackers provided by media outlets and health-related websites (e.g., the CDC [14] and states' Department of Public Health). Additionally, unlike past work on COVID-19, which presumes a static relationship between the presence or absence of trust and people's behaviors [56], we examine the dynamic relationship between information practices via ICTs and STSs, and the evolving formation of trust and distrust. Moreover, little work that studies the intersection of trust and ICTs and STSs has focused on traditionallymarginalized populations, like low-SES communities, who have been disproportionately impacted by the pandemic. Our research seeks to address these research gaps. 


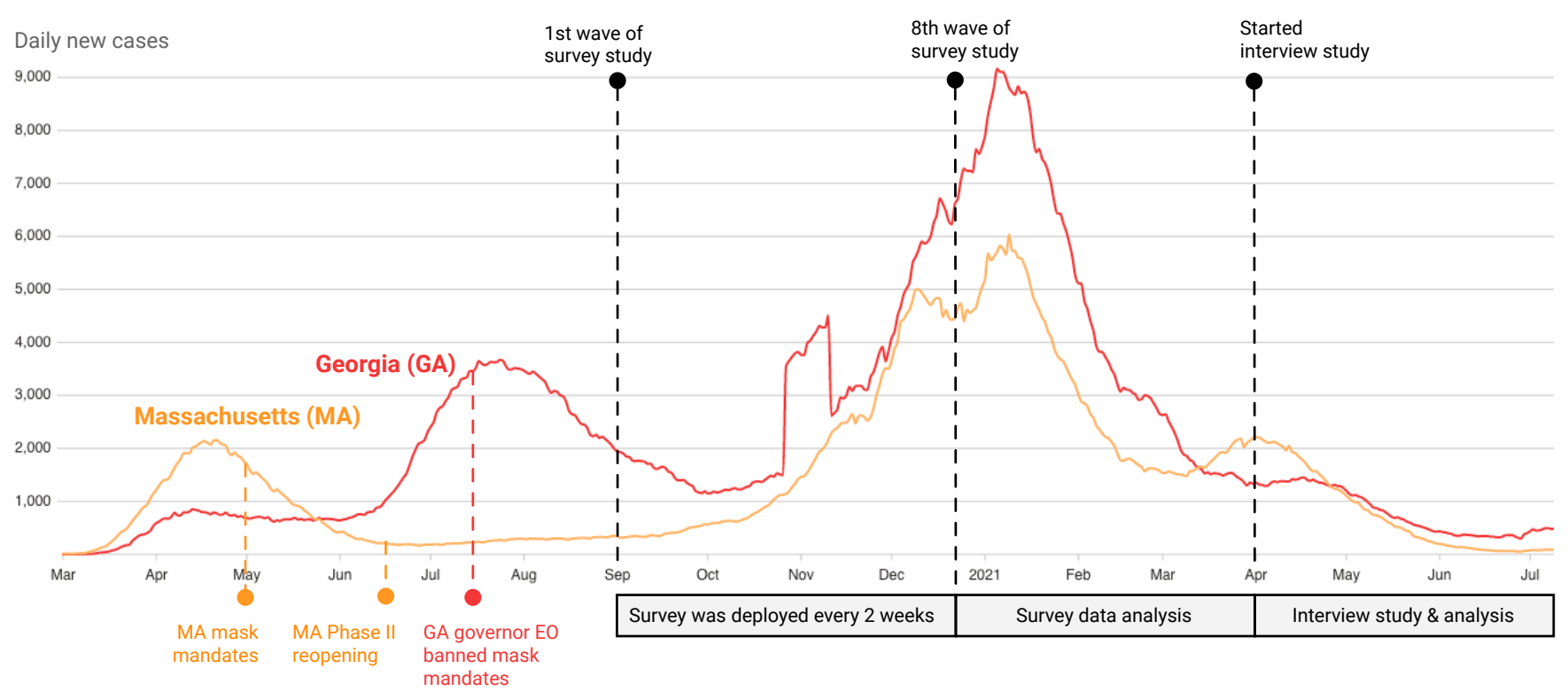

Figure 1: An overview of COVID-19 situations of Massachusetts (MA) and Georgia (GA) in the United States: This figure shows a brief timeline with several key dates. During our study design and deployment period, MA and GA had shown different trends and patterns during the pandemic (e.g., confirmed cases/deaths), as well as different public health policies during COVID19. For example, the MA Governor issued an order requiring the use of masks or face coverings in public places on May 1 , 2020. However, GA has never issued mask mandate policy during COVID-19. Data sources: Cases data is from Johns Hopkins University CSSE [40]; policy events are drawn from National Governor's Association [59].

\section{METHOD}

This paper is part of a larger project examining information practices and attitudes during COVID-19 and how these practices and attitudes vary across populations. We specifically focus on populations that have experienced disproportionate COVID-19 morbidity, mortality, and hardship, such as low-SES adults [2]. Upon approval from the Institutional Review Board at our institution, we conducted a mixed method study that included a longitudinal survey with 177 adults and interviews with 21 participants who completed all waves of the survey study. The use of mixed methods enables triangulation, an approach to analyze results from the same study using different methods of data collection [41]. Triangulation helps enhance the validity of our research and creates a more in-depth picture of understanding the social and technological dimensions of people's information practices amidst the COVID-19 pandemic. Below we detail the methods used in the survey (subsection 3.1) and interview (subsection 3.2) components of this project.

\subsection{Survey Study}

We conducted a longitudinal survey from September 1, 2020 to December 21, 2020. The focus of our survey was to examine people's information seeking behaviors amidst COVID-19. Given the rapid progression of the pandemic in 2020, we expected that individuals' information practices would also change over time, which includes shifts in the platforms used to obtain COVID-19 information as well as the information received. Further, while other studies have shown how demographic differences exist in information practices [30,92], our work seeks to identify how these differences relate to information about COVID-19. Therefore, our survey design was guided by the following overarching research questions: 1) how do people's information practices change over time? and 2) what demographic variations exist in information practices, and how do these variations differ in relation to trust and distrust towards COVID-19 information?

In the following sections, we describe in detail the survey study procedures, survey questions and measurements, and analyses.

3.1.1 Survey Study Procedures. We conducted several rounds of pilot testing to develop and refine the survey. First, we conducted multiple rounds of informal pilot testing within the research team and through our family and friend networks. Upon revision based on their feedback, the survey was then formally pilot tested with 40 respondents via Prolific ${ }^{2}$; each participant was compensated with $\$ 4$ for their time. Feedback and responses from the pilot tests were used to iterate upon and develop the final survey.

Our study inclusion criteria were as follows: participants were adults (aged 18+) who live in Massachusetts (MA) or Georgia (GA) and had a 2019 household income of less than $\$ 100,000$. We chose this income threshold to narrow our focus to low- and middlesocioeconomic populations; doing so enabled us to investigate how information practices and attitudes vary between these two income levels. Respondents were also classified into two income groups (low- and middle-income) ${ }^{3}$ based on the 2020 U.S. Federal Poverty

\footnotetext{
${ }^{2}$ https://prolific.co/

${ }^{3}$ Respondents were classified as low-income if their adjusted household income was less than or equal to $185 \%$ of the FPL, following the eligibility guidelines for federal aid programs [89].
} 
Table 1: Sample demographic characteristics of our survey respondents and interview participants.

\begin{tabular}{llrr}
\hline \multirow{2}{*}{ Demographic } & Response Options & Interview Participants & Survey Respondents \\
& & $(\mathrm{N}=21)$ & $(\mathrm{N}=177)$ \\
\hline \multirow{2}{*}{ Gender } & Female & $14(66.7 \%)$ & $111(62.7 \%)$ \\
& Male & $6(28.6 \%)$ & $60(33.9 \%)$ \\
& Prefer not to say/self-describe & $1(4.8 \%)$ & $6(3.4 \%)$ \\
\hline \multirow{2}{*}{ Age } & $18-24$ & $3(14.3 \%)$ & $29(16.4 \%)$ \\
& $25-34$ & $7(33.3 \%)$ & $50(28.2 \%)$ \\
& $35-44$ & $6(28.6 \%)$ & $51(28.8 \%)$ \\
& $45-54$ & $3(14.3 \%)$ & $28(15.8 \%)$ \\
& $55+$ & $2(9.5 \%)$ & $19(10.7 \%)$ \\
\hline \multirow{2}{*}{ Race } & Asian & $2(9.5 \%)$ & $13(7.3 \%)$ \\
& Black & $7(33.3 \%)$ & $51(28.8 \%)$ \\
& Hispanic & $3(14.3 \%)$ & $11(6.2 \%)$ \\
& White & $9(42.9 \%)$ & $99(55.9 \%)$ \\
& Other/Mixed & & $3(1.7 \%)$ \\
\hline Education & Less than Bachelor Degree & $7(33.3 \%)$ & $114(64.4 \%)$ \\
& Bachelor Degree and Higher & $14(66.7 \%)$ & $63(35.6 \%)$ \\
\hline Income group & Low & $6(28.6 \%)$ & $80(45.2 \%)$ \\
& Middle & $15(71.4 \%)$ & $97(54.8 \%)$ \\
\hline State & GA & $13(61.9 \%)$ & $78(44.1 \%)$ \\
& MA & $8(38.1 \%)$ & $99(55.9 \%)$ \\
\hline
\end{tabular}

Level (FPL) Guidelines [88]. To enable exploration into the effects of information, which is expected to be correlated with the state of the pandemic, we purposefully focused on two regions which differed in policy approaches [59] and incidence patterns [40] at the beginning of the study (see Figure 1). Further, our local knowledge of these regions ensured that locally-relevant questions were included in interviews and surveys.

Following the inclusion criteria, participants were recruited into the longitudinal study through the Qualtrics online research panel service [63]. Qualtrics recruited participants from a nationally representative panel. This service helps in the recruitment process by advertising the survey study to an existing pool of respondents. These respondents have agreed to be contacted by the Qualtrics company regarding survey opportunities. Respondents complete surveys in exchange for incentives issued by the company. ${ }^{4}$

A total of 329 participants completed and passed our quality checks in the first survey and were invited to participate in subsequent surveys ${ }^{5}$. Each survey was deployed every two weeks and each deployment is denoted in this paper as a wave (i.e., Wave 1, Wave $2, \ldots$, Wave 8 ). In total, 177 participants completed all 8 waves of the surveys with an average of $92 \%$ retention rate per wave. ${ }^{6}$

3.1.2 Survey Questions and Measurements. We utilized survey questions that were focused on individuals' information practices during COVID-19 and their level of trust regarding the information

\footnotetext{
${ }^{4}$ Incentive structures were set by the company and not the research team, and Qualtrics was unable to disclose the compensation details to us.

${ }^{5}$ Quality checks include $98 \%$ survey completion rate.

${ }^{6}$ Participants who dropped-out at any point of the survey were not included in the analysis since we cannot fully assess the changes in their survey responses.
}

sources. Here, we outline the questions and measures used within the survey for the scope of this paper: ${ }^{7}$

(1) How often did you use the following platforms; i) radio, ii) television, iii) print newspaper, iv) internet-connected devices, to get information or news about the coronavirus? (1="never", 2="rarely/once a week", 3="sometimes/2-3 times a week", 4="often/4-6 times a week", 5= "every day")

(2) Please choose social media platforms you used the most to get information or news about the coronavirus; i) Facebook, ii) YouTube, iii) Twitter, iv) Reddit, v) I did not use social media to get coronavirus information ( $0=$ "no", $1=$ "yes") ${ }^{8}$

(3) I did not get coronavirus information from websites ( $0=$ "no", $1=$ "yes") 9

(4) On a scale of 0 (do not trust at all) to 10 (complete trust), how much do you trust the following individuals or organizations in providing coronavirus information? i) family members, ii) friends, iii) Centers for Disease Control and Prevention (CDC), iv) Dr Anthony Fauci (Director of the National Institute of Allergy and Infectious Disease), v) Local government agencies and officials

(5) About how often did you get the information about COVID19 number of cases and/or deaths (1="never", 2="rarely/once a week", 3="sometimes/2-3 times a week", 4="often/4-6 times a week", $5=$ "every day")

\footnotetext{
${ }^{7}$ Checkboxes were provided for question (2) and (3).

${ }^{8}$ These social media platforms were selected as they were cited as the primary information sources for COVID-19 related information and news [42].

${ }^{9}$ News apps did not count as news websites.
} 
(6) How likely do you think it is that "authorities are intentionally reporting inaccurate rates of coronavirus infections and deaths in the United States"? (1="extremely unlikely", $2=$ "unlikely", $3=$ ="maybe/not sure", 4="likely", 5="extremely likely”)

3.1.3 Survey Analysis. To allow for characterization and comparison of change in the variables of interest over time, we performed linear mixed-effects regression to analyze our survey data [26]. To analyze if a variable (i.e., social media platform usage) changes over time, we treated subjects (i.e., respondents ID) as random effects and time (i.e., survey wave) as fixed effects. When analyzing variables association, the variable of interest (i.e., frequency of receiving information about number of cases/deaths) is treated as fixed effects, whereas both subjects and time are treated as random effects. All statistical analyses were performed in $\mathrm{R}$ version 4.1.2.

\subsection{Interview Study}

Our survey data analysis was conducted from January to April 2021. To further investigate the trends in our survey data, we then conducted follow-up semi-structured interviews from April 2021 to July 2021 using Zoom ${ }^{10}$ (an online meeting tool).

3.2.1 Participant Recruitment. We reached out to all survey respondents who expressed interest in participating in a follow-up interview via email and text message, of which 30 participants agreed to participate, and 21 participants completed the interview ( 9 participants did not show up or cancelled the interview session). Table 1 provides an overview of the demographic information for our survey respondents and interview participants.

3.2.2 Interview Study Procedures. We analyzed each interview participants' survey data from each survey wave to identify patterns and trends for each question listed in subsubsection 3.1.2. During the interview, participants were asked to further elaborate on their information behaviors throughout the pandemic, allowing us to explore, in detail, the patterns and trends shown in the survey results. The interview sessions were recorded for analysis purposes and lasted for approximately 80 minutes. At the end of the interview session, each participant was compensated with a $\$ 25$ gift card.

3.2.3 Interview Data Analysis. All interview sessions were transcribed verbatim. We used the General Inductive Approach [85] to guide the thematic analysis of the transcripts. We first read the transcripts closely to get an initial understanding of the concepts that arose in the interview data. Then, we analyzed the transcripts and created low-level codes to label concepts in the data, clustering related low-level codes to achieve high-level themes. Throughout the analysis, all authors discussed and refined the emerging themes regularly.

By analyzing both the survey and interview data, we seek convergence and complementarity-two common types of triangulation [41]. Convergence indicates a strong degree of overlap and accuracy between the data sets collected using quantitative and qualitative methods, whereas complementarity builds a richer picture of the research results by allowing the results from different methods to inform each other [41].

\footnotetext{
${ }^{10}$ https://zoom.us/
}

\section{SURVEY RESULTS}

In this section, we present results from 177 survey respondents who completed all 8 waves of the survey. For the scope of this paper, we focus on the patterns and trends of respondents' information consumption via a variety of information platforms, as well as their use of and level of trust in information sources in subsection 4.1. We also report results regarding the information exposure and level of distrust regarding COVID-19 data in subsection 4.2. Note that in reporting our survey results and interview findings, we use "COVID-19 data" in a very specific context, referring to the number of COVID-19 cases and deaths. While the survey results provided some facts about respondents' trust and its association with their information practices during COVID-19, the results also prompted a series of questions that necessitated further investigation. Therefore, at the end of each subsection, we summarize questions (labeled with $\mathbf{Q}$-) that we sought to examine in our interview study (section 5). Note that we use the term "respondents" for survey data and "participants" for interview data.

\subsection{Use of Platforms and Sources for Getting COVID-19 Information \& Assessment of Trust Over Time}

In this subsection, we report the survey results regarding the overall information consumption via different information platforms (e.g., television, radio, newspaper, internet), as well as participants' use of and level of trust in different information sources. Figure 2a shows the overall trend of various information platforms utilized to obtain COVID-19 information during our survey period. Overall, our survey results suggest a high use of technology for receiving COVID-19 information. Yet, the use of the internet to obtain COVID19 information significantly decreased over time $(\beta=-0.019, p<$ $0.001)$. In contrast, the use of other traditional media platforms (e.g., television, radio, and print newspaper) to obtain COVID-19 information saw no significant change over time.

In terms of the use of social media and websites for COVID-19 information consumption (see Figure $2 b$ ), our survey results show that there was a significant decrease in social media utilization over time $(\beta=-0.025, p<0.001)$, referring to the slope of the regression line. While the types of social media platforms that were utilized varied from Facebook, YouTube, Twitter, and Reddit (see Figure 2c), we note a significant decrease in Facebook usage over time $(\beta=$ $-0.028, p<0.01$ ). Similar to social media, the use of websites for gaining COVID-19 information also significantly decreased over time $(\beta=-0.016, p<0.001)$, where the lowest usage was reported in Wave $5(62 \%)$.

Nonetheless, our survey results also suggest trust in personal networks and scientific communities were associated with income and education groups. Of note, income and education in our study are independent based on chi-square tests $\left(\chi^{2}=103.66, p<0.001\right)$. Specifically, respondents with middle income and at least a bachelor's degree reported significantly higher trust in Dr. Fauci than respondents with low income $(\beta=0.133, p<0.001)$ and without a bachelor's degree $(\beta=0.072, p<0.001)$. Yet, those with middle income trusted their family members $(\beta=-0.053, p<0.05)$ and friends $(\beta=-0.071, p<0.01)$ significantly less than those with low income. No significant differences were found in the trust of the CDC and 

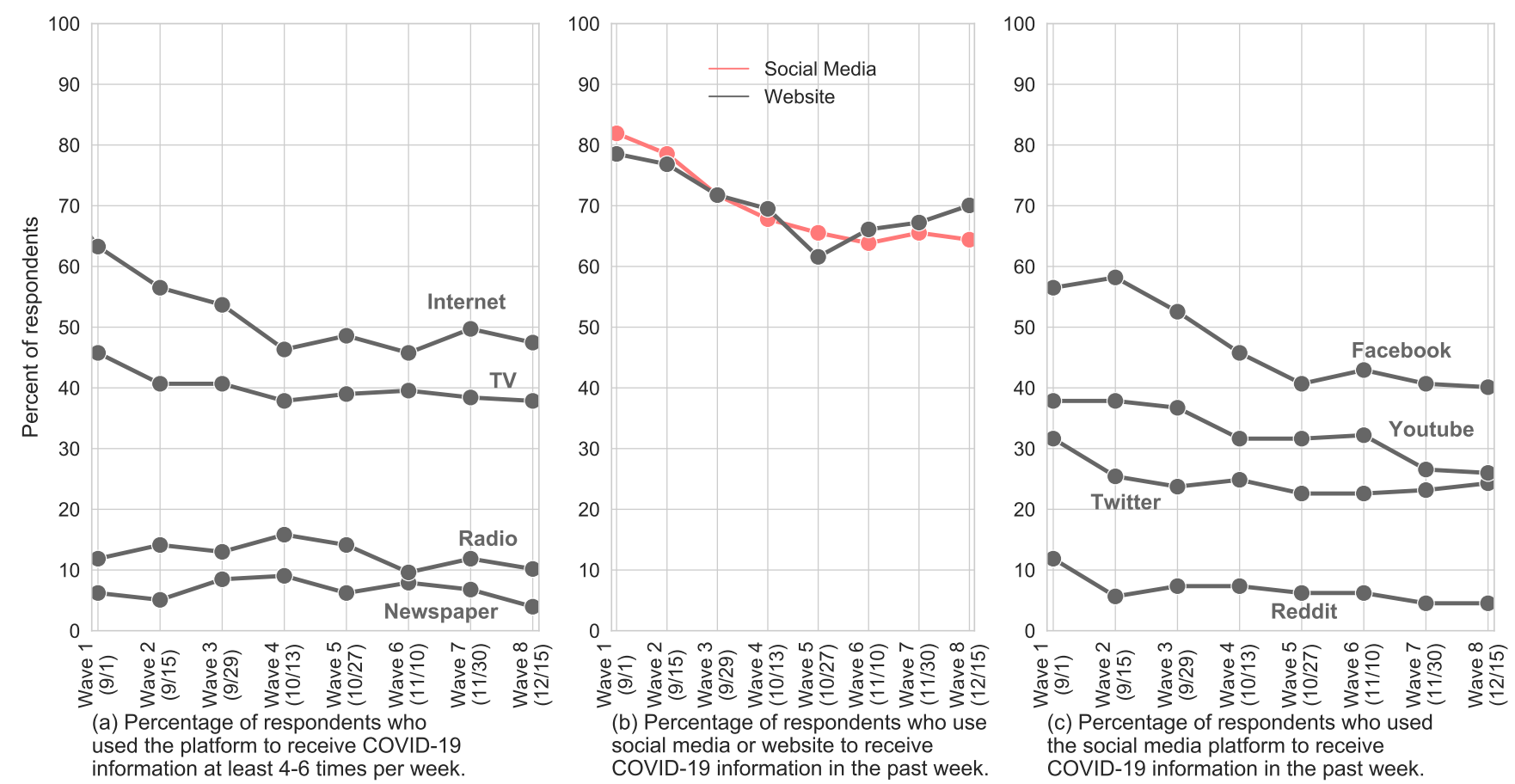

Figure 2: The use of information platforms for COVID-19 information consumption during our survey period.

Table 2: Multi-level regression results on the effect of income and education on information practices during COVID-19. (Significance: $* p<0.05, * * p<0.01, * * * p<0.001$ )

\begin{tabular}{llll}
\hline & & $\begin{array}{l}\text { Middle income } \\
\text { (vs. Low income) }\end{array}$ & $\begin{array}{l}\text { Bachelor and higher } \\
\text { (vs. Less than bachelor) }\end{array}$ \\
\cline { 2 - 4 } Dependent Variables & & $\beta$ (Std. Error) & $\beta$ (Std. Error) \\
\hline Platforms utilized to get COVID-19 info & Internet & $0.054(0.027)^{*}$ & $0.012(0.020)$ \\
& TV & $-0.004(0.026)$ & $-0.057(0.019)^{* *}$ \\
& Radio & $-0.027(0.018)$ & $-0.038(0.013)^{* *}$ \\
& Newspaper & $0.014(0.003)$ & $-0.034(0.010)^{* * *}$ \\
& Website & $0.126(0.024)^{* * *}$ & $0.094(0.018)^{* * *}$ \\
& Social media & $0.081(0.024)^{* * *}$ & $-0.056(0.018)^{* * *}$ \\
\hline Specific social media platform & Facebook & $-0.052(0.026)$ & $0.024(0.019)$ \\
& Twitter & $-0.001(0.023)$ & $0.063(0.017)^{* * *}$ \\
& Reddit & $-0.026(0.013)$ & $-0.002(0.010)$ \\
& YouTube & $-0.116(0.025)^{* * *}$ & $-0.050(0.018)^{* *}$ \\
\hline COVID-19 information source & Family & $-0.053(0.027)^{*}$ & $-0.021(0.020)$ \\
& Friends & $-0.071(0.025)^{* *}$ & $0.011(1.836)$ \\
& CDC & $0.040(0.026)$ & $0.028(0.019)$ \\
& Dr Fauci & $0.133(0.025)^{* * *}$ & $0.072(0.019)^{* * *}$ \\
\hline Frequency of getting information about COVID-19 data & Local government & $0.030(0.026)$ & $-0.014(0.019)$ \\
\hline Distrust in authorities regarding COVID-19 data & $-0.468(0.073)^{* * *}$ & $-0.163(0.055)^{* *}$ \\
\hline
\end{tabular}




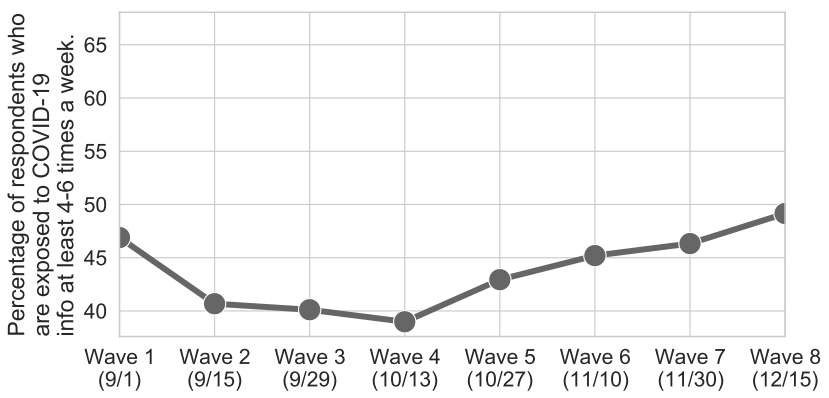

Figure 3: The change in the percentage of participants who received information about COVID-19 data at least 4-6 times a week.

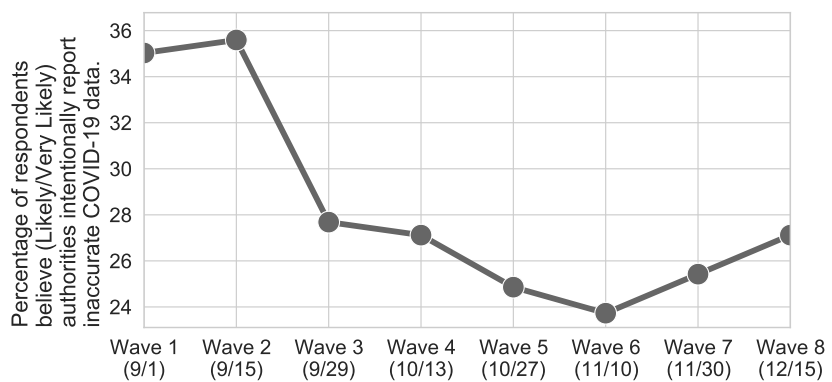

Figure 4: The change in distrust in authorities regarding COVID-19 data was assessed by asking respondents if they believe the authorities intentionally report inaccurate COVID-19 data.

local government for providing COVID-19 information. Additionally, respondents with middle income received significantly more information via the internet $(\beta=0.054, p<0.05)$, including from websites $(\beta=0.126, p<0.001)$ and social media $(\beta=0.081, p<$ 0.001).

There is no significant difference in the information exposure to COVID-19 data among income groups, but respondents with at least a bachelor's degree received significantly less information about COVID-19 data than those without a bachelor's degree $(\beta=-0.252$, $p<0.05)$. Distrust in authorities regarding COVID-19 data reporting was found to be significantly less among those with higher incomes ( $\beta=-0.468, p<0.001)$ and higher education $(\beta=-0.163, p<0.01)$. Additional characteristics of respondents' information practices are reported in Table 2.

Summary: Our survey results indicate a high utilization of the internet to obtain COVID-19 information. However, this utilization decreased over time. Additionally, while the use of traditional media platforms (e.g., radio, television, and print newspaper) remained consistent, the use of social media for COVID-19 information consumption significantly decreased over the course of our survey period. These results motivated us to further investigate the following questions through our interview study: Qa. What drove our respondents to reduce their engagement with COVID-19 information through the internet, and $\mathbf{Q b}$. Does trust play any role in explaining the decreasing trend in users' social media engagement for the consumption of COVID-19 information?

\subsection{Information Consumption \& Distrust Regarding COVID-19 Data}

In this subsection, we report the survey results of information consumption regarding COVID-19 data, as well as the extent to which individuals distrust authorities regarding this data.

Figure 3 shows the overall trend of information consumption about COVID-19 data among our survey respondents. Our analysis results suggest no significant change in the frequency of obtaining this information over time. However, the belief that authorities intentionally reported inaccurate COVID-19 data decreased significantly over time $(\beta=-0.071, p<0.001)$, as shown in Figure 4, suggesting a decrease in distrust over time. In wave 1, 62 out of 177 (35\%) respondents reported "likely" and "extremely likely" in their belief that authorities intentionally reported inaccurate data, while 48 out of the $177(27 \%)$ respondents continued to believe that the reports were inaccurate through Wave 8.

The belief that authorities intentionally reported inaccurate data was also found to be negatively associated with the frequency in which individuals received information about COVID-19 data ( $\beta$ $=-0.114, p<0.001)$. In other words, the more information that individuals received regarding COVID-19 data, the less likely they were to believe that authorities intentionally reported inaccurate data. Simultaneously, the less information that individuals received, the more likely they were to believe that authorities were reporting inaccurate data.

Summary: Our survey results show that there is an association between an individual's information consumption regarding COVID-19 data and their distrust behaviors. Moreover, we found that over time, an individual's level of distrust in the reporting practices of authorities decreased significantly between September and December of 2020. Therefore, in the interview study, we aimed to explore two additional questions: Qc. In what ways is our participants' distrust associated with their information consumption? and Qd. Why does respondents' distrust in authorities decrease over time with regards to the sharing practices of COVID-19 data?

\section{INTERVIEW FINDINGS}

In this section, we report our qualitative analysis results captured in the interview study, triangulated with our survey results. We focus on ways that participants articulated trust and distrust in relation to their information practices amidst COVID-19. This section also seeks to answer various questions that arose from the survey results.

To allow for a situated examination, we summarized participants' information behavior, highlighting how they received COVID-19 information and what sources they utilized (e.g., personal networks, scientific communities, local government, and social media ${ }^{11}$ ) in Table 3. We also summarized their information consumption and their level of distrust regarding COVID-19 data in Figure 5.

\footnotetext{
${ }^{11}$ In Table 3: "All-wave use" = used social media for getting COVID-19 information across all waves; "All-wave non-use" = did not use social media at all across all waves; "Stopped use" = used social media during the first 1-2 waves at most, but then stopped using during the rest of the waves; "Sometimes use" = used social media irregularly during the waves (excluding the "stopped use" behaviors).
} 
Table 3: An overview of our 21 interview participants' information practices, specifically indicating the sources they used to obtain COVID-19 information.

\begin{tabular}{ccccccc}
\hline PID & Family & Friends & CDC & Dr. Fauci & Local Gov & Social Media \\
\hline P01 & Yes & Yes & Yes & Yes & Yes & Sometimes use \\
P02 & Yes & Yes & Yes & Yes & Yes & All-wave use \\
P03 & No & Yes & Yes & Yes & Yes & All-wave non-use \\
P04 & No & No & Yes & Yes & Yes & All-wave use \\
P05 & Yes & Yes & Yes & Yes & Yes & Stopped use \\
P06 & No & Yes & No & No & No & Stopped use \\
P07 & No & Yes & Yes & Yes & Yes & Stopped use \\
P08 & Yes & Yes & Yes & Yes & Yes & Stopped use \\
P09 & Yes & Yes & Yes & Yes & Yes & Sometimes use \\
P10 & Yes & Yes & Yes & Yes & Yes & Sometimes use \\
P11 & Yes & Yes & Yes & Yes & Yes & Sometimes use \\
P12 & Yes & Yes & Yes & Yes & Yes & Stopped use \\
P13 & Yes & Yes & Yes & Yes & Yes & All-wave use \\
P14 & Yes & Yes & No & No & No & Stopped use \\
P15 & Yes & Yes & Yes & Yes & Yes & Stopped use \\
P16 & Yes & Yes & Yes & Yes & Yes & All-wave use \\
P17 & No & Yes & Yes & Yes & No & All-wave use \\
P18 & No & Yes & Yes & Yes & Yes & All-wave use \\
P19 & Yes & Yes & Yes & Yes & Yes & All-wave use \\
P20 & Yes & Yes & Yes & Yes & Yes & Sometimes use \\
P21 & No & Yes & No & No & Yes & All-wave use \\
\hline
\end{tabular}

In the remainder of this section, we first examine what drives our participants to reduce their engagement with various information platforms (e.g., social media) to obtain COVID-19 information, including the ways in which trust plays a significant role in explaining this diminished usage in subsection 5.1. We then investigate how participants articulate their trust and distrust regarding COVID-19 data in subsection 5.2.

\subsection{Trust in Social Media}

We first provide some brief context to help explain the reduced utilization of the internet to consume COVID-19 information (to answer Qa). Then, we specifically discuss the role that trust plays in explaining the diminished social media engagement amongst participants (to answer $\mathbf{Q b}$ ).

According to our participants, a lack of time $(n=6)$ is one of the major reasons for the diminished use of the internet for COVID19 information consumption. Since some of our participants were from low-SES households, they mentioned that they did not have the privilege to work from home during the pandemic. Many participants had gone back to work in person during the reopening phase in summer 2020. Adapting to their new lifestyle, low-SES participants (e.g., P1, P11, P14, P20) indicated that they had other things that "started to consume their life", such as going back to work in person. Furthermore, a second reason for the decrease in COVID-19 information consumption lies in information fatigue $(\mathrm{n}=6)$. Information fatigue is the subjective feeling of tiredness as a result of being exposed to very similar messages and news for an extended period of time [72]. For example, our participants mentioned that they would look at COVID-19 data and related information

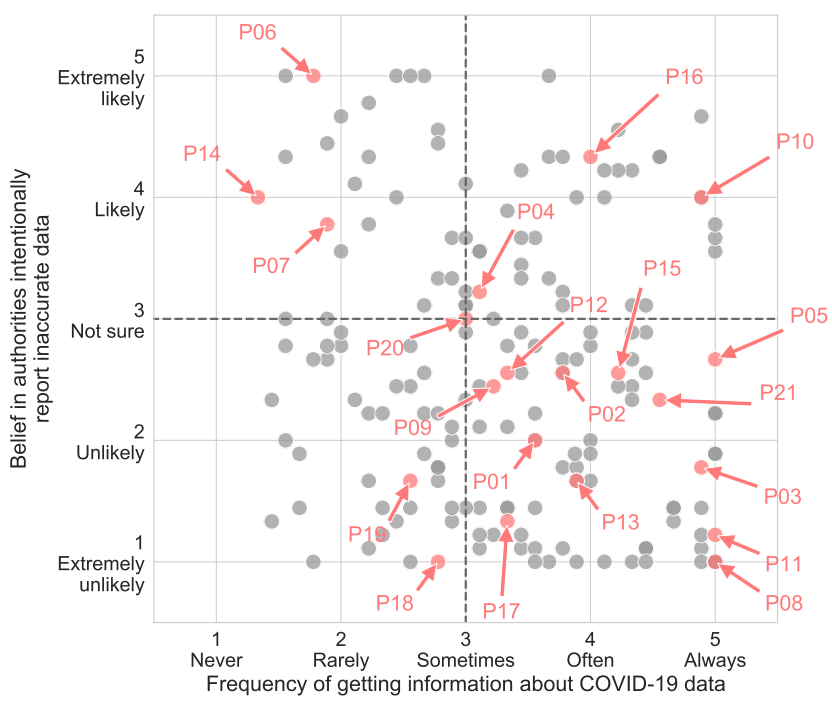

Figure 5: The relationship between the frequency of getting information about COVID-19 data (e.g., cases/deaths) and the belief in authorities intentionally reporting inaccurate COVID-19 data. Each data point represents the average of values across all waves. represents interview participants' data with an arrow pointing to the participant ID (e.g., $\leftarrow$ PID); represents all 177 survey respondents' data (note some data points overlapped). 


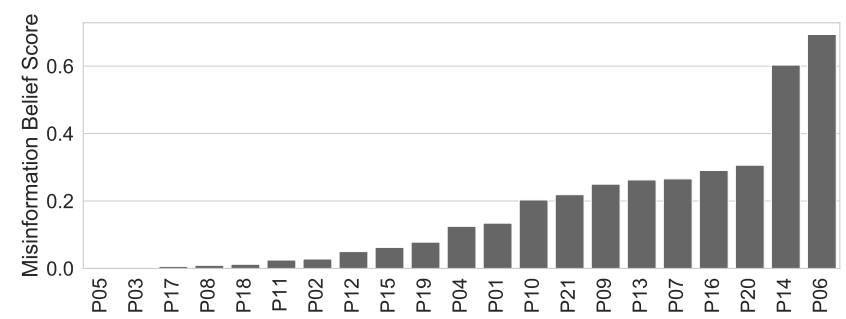

Figure 6: How likely our participants trust misinformation, ranked from lowest to highest. Misinformation Belief Score is the sum of their selection across a list of 5-point (Never $=0$, Unlikely=1, Not sure =2, Likely=3, Extremely Likely=4) questions over all 8 waves divided by the score if the participant always select Extremely Likely. Questions include 1) The coronavirus does not exist, 2) The coronavirus was purposefully created and released as part of a conspiracy, 3) Hydroxychloroquine Chloroquine, and Azithromycin can be used as a treatment for the coronavirus, 4) Diluted bleach can be used as a treatment for the coronavirus, 5) The coronavirus is caused by $5 G$ technology, 6) The thread of the coronavirus has been exaggerated by political groups who want to damage President Trump, 7) The coronavirus is being use to force a dangerous and unnecessary vaccine on Americans, and 8) The coronavirus outbreak is a populationcontrol scheme developed by Bill Gates.

more often at the beginning of the pandemic, but then "after a while people get tired" and "did not have the mental energy to keep up with" this information as time went by.

However, we found that a decline in trust accounted for the largest change in social media use for COVID-19 information consumption. Below we report findings regarding how our participants articulated their trust and distrust in social media.

5.1.1 An Inability to Change Others' Trust Leads to Non-use. Our participants were varied in their use of social media to obtain COVID-19 information, as shown in Table 3. We found that 8 participants used social media across all waves; 7 participants used social media during the early waves but then stopped using it; 5 participants sometimes used social media (a mix of use and non-use across waves); and 1 participant did not use social media at all during any of the waves.

One of the most influential factors that explained the diminished social media usage for COVID-19 information consumption is the avoidance of tension and conflict with others on social media networks, especially amongst family and friends. Further, these tensions were grounded in issues of trust. Our participants described various examples of ways in which their views conflicted with the views of their social media contacts (e.g., friends and families on Facebook). For example, P15 said,

"I have a Facebook account, but I do it because I have family around the country [and] outside US, and I follow them. But I'm not going to get into a debate over it, because [my Facebook contacts] have very strong opinions, and they will lash out... Do you really want to get in a public fight with your family [on Facebook]?"

(P03, 55-64, Female, Associate Degree, Democrat)

We noticed that P15's survey results showed that she only used social media for COVID-19 information during the first wave of our study but did not use it throughout the rest of the study period, as shown in Table 3. It is worth noting that she did not begin using other platforms for COVID-19 information consumption once she stopped using social media.

The tension that our participants experienced while interacting with social media during the pandemic was mostly due to conflicting perspectives and different assessments of COVID-19 misinformation. Participants felt a sense of inability to modify others' misbelief. Failing to change others' beliefs frustrated our participants. For example, P18 explained,

"My perspective is different from my family's perspective in a lot of different ways, and actually I was yelling at my parents and my sister... my mom's side of the family is super religious, and on their social media, they were just complaining about the government ... not even just saying it was fake, and all this other stuff and upset because they were in New York. And you know how New York shut everything down. So, they were saying it was the government's way of removing religion. I'm like [this is] just bogus stuff which is crazy, but everyone has their own opinions... I don't know what to do and argue for..." (P18, 25-34, Female, Graduate Degree, Democrat)

As one may imagine, arguments and conflicting views on social media did occur prior to the pandemic. However, according to our participants, it was COVID-19 that "intensified" and "reignited" the tension and conflict on social media. For example, P11 mentioned that she had never experienced such feelings of frustrations on social media. She told us that "there were not too many people like who have been crazy prior to the pandemic in my group". Yet, during COVID-19, she indicated that she was getting angry about her Facebook contacts' posts. She elaborated,

"I used to be on Facebook. I don't search much, but one or two items I search, I get the alert about it like somebody posted. And sometimes that used to make me pissed off because the people have different thoughts and all. So I tried not to watch this stuff on Facebook because it makes me angry because people knows everything going around and they don't care... They make fun of the people who are wearing masks or taking precautions, so I try to avoid not to talk much about it on this Facebook stuff because it's a crazy and a lot of misinformation is going to make you pissed off. It's going to be make you more angry because if I'm going to talk, it's going to mess up somewhere my relationship. I try not to discuss this stuff on Facebook, actually intentionally avoiding Facebook feeds [about COVID-19]... I don't want to go and fight with [my social media contacts] because the people who are not going to understand, they're never gonna understand. If you try to make them understand, they would say it's like you are the racist..." (P11, 25-34, Female, Bachelor Degree, Independent)

Additionally, some participants $(n=4)$ mentioned that their family members who trusted misinformation on social media also had a 
tendency to trust other sources of misinformation outside of social media. For example, P02 mentioned,

"like with my parents are like very religious... So my dad trust information from the church and like priests will go like oh don't use this vaccine, like this vaccine uses like abortion cells... so I'm like 'please, my parents' [I asked my parents to not to trust the vaccine misinformation], at the same time I'm just little bit of anger... I feel like I am in a population where misinformation spreads easily within our community." (P02, 18-24, Non binary, Bachelor degree, Democrat)

These quotes from P18 and P02 suggest a generational gap in information behaviors within social media. By generational gap, we mean the disparity of beliefs and information behaviors between distinct age groups or generations. In other words, members from a younger generation in our study (like P18, P02) exhibited different perspectives, beliefs, and decisions regarding information online as compared to the older individuals in their lives, like their family members. Our interview data aligns with prior research that indicates that older generations have higher engagement with fake news on social media than younger generations [7].

5.1.2 Using But Not Trusting Social Media. While our survey results suggested a significant decrease in social media usage, there was still a large portion of survey respondents who utilized social media for COVID-19 information. However, during our interview study, we found that most participants did not trust social media for COVID-19 information.

Our findings suggest that many of our interviewees believed that they could distinguish misinformation from factual information on social media. The majority of our participants $(n=18)$ felt as though they could trace information back to its original source to determine if it is credible or not. Amongst the people who continued to use social media, most explained that although they were exposed to this information, they did not necessarily trust it. For example, P12 mentioned,

"I have a Facebook account... But I wasn't using it to make decisions. It's a little unreliable. I feel like it's not representative of people's actual lives or lifestyles. I don't feel like it's necessarily something to be trusted. I value the personal communication, [and] I'd rather talk to someone about what's going on in life than post it for everyone to see on the social media." (P12, 18-24, Female, Bachelor Degree, Independent)

Like P12, many other participants $(\mathrm{n}=10)$ described situations where they encountered COVID-19 information on their social media feeds, indicating that they disregarded or tended to "not rely on" this information. "Not relying on" indicates that participants neither reached an adequate trust level for information from social media nor did they use it to make decisions. As such, it is important to differentiate the "use" of social media for information from the reliance on it. For individuals who can distinguish factual information from false information on social media, misinformation may not necessarily impact their decisions and behaviors.

While some participants in our study felt they were able to identify misinformation and maintained that their social media use did not negatively impact their behavior, such self-reported assessments might be contradicted by more objective evaluations of individuals' ability to distinguish between true and false information [95]. In such cases, this discrepancy would reveal an apparent disconnect between individuals' perceived ability to discriminate between fake and factual information versus their actual ability.

However, interestingly, by triangulating with our survey data, we find that interview participants who indicated that they could identify misinformation (e.g., P02, P08, P11, P12) were among the people who were indeed the least likely to believe misinformation according to Figure 6. These findings highlight the value of combining subjective and objective assessments of people's ability to identify misinformation, as well as the value of ensuring that people's personal assessments of their abilities are not discounted.

5.1.3 Trustee Shifts: Trusting Information on Social Media or Trusting the Social Media Platform Itself? Social media websites are information platforms where various user-created information is assembled. Users typically need to sort through posts to determine the trustworthiness of each piece of information. In this scenario, the individual pieces of information (e.g., an article, a video, or a social media feed or tweet) are considered the trustee (i.e., the trusted party). However, for some participants, the focus was on the trustworthiness of the social media platform(s) itself, rather than the trustworthiness of the information within the platform. In essence, participants' past experiences with misinformation on social media platforms has produced a generalized distrust for these platforms in their entirety. As such, the trustee shifts from the pieces of information to the social media platforms themselves. Once this occurs, distrust is ingrained into the trustor (i.e., information consumer), which consequently makes rebuilding trust more challenging. Here, we discuss the mechanism through which the trustee shifts from pieces of information to the social media platform(s). We also reflect on why it is important to unpack this trustee shift, as well as its implications in section 6.

Many participants $(n=9)$ indicated that they did not trust information on social media because they realized that social media was composed of a mixture of true and false information. P09 explained,

"There are lots of things that go on with social media. By the time that I decided many years ago to tune out of that, I was aware that there was a mixture of real information and fake information. And some people find it entertaining, but I do not, so I didn't go there. So that gave me insight not to trust social media." (P09, 35-44, Female, Graduate Degree, Democrat)

And yet, our participants were afraid that misinformation may unconsciously impact them. Some $(n=2)$ pointed out that people tend to forget to verify the credibility of the source when they obtained information and were concerned about unconsciously falling into the "trap" of misinformation. P15 explained, "social media is so unreliable... It's just something that popped up that was fun to use to talk to your friends, [but] it's not supposed to be a news source or something reliable... [Potentially fake news] was interesting. But you stop thinking when you read it and forget the source..." (P15, 35-44, Female, Graduate Degree, Independent) 
Given how quickly people ingest content, social media users also regularly encounter harmful misinformation of which they might not be aware. A large body of work provides suggestions for social media users, identifying how to recognize misinformation by scrutinizing its source [23, 65]. Yet, as our participants described, human beings do not always act rationally or behave as planned.

Furthermore, the mixture of trustworthy information and misinformation on social media can invoke fear in people. As participants experienced challenges in spotting and differentiating between the "facts" and misinformation, their concerns and fearfulness towards social media was deepened. For example, our participants explained how their negative experiences with social media misinformation, as well as their deep concerns about believing misinformation, led to their non-use of social media for COVID-19 information,

"It is a lot of misinformation out there on social media, and it's hard to discern it, but that kind of stuff will make me anxious and afraid. It will make me nervous and put me in a place emotionally I don't want to be so [I didn't use social media for getting COVID-19 information]" (P20, 35-44, Male, Associate Degree, Republican)

For some participants (e.g., P09, P15, P16, P20), their perception of social media as a mixing pot of true and false information, as well as the uncertainty in their ability to discern this misinformation, generates a sense of insecurity and fearfulness among users in the social media platforms. These negative attitudes collectively contribute to their distrust in the platforms. Indeed, this interpretation of distrust is in line with work that suggests trust is an affective attribute [39]. Distrust carries affective orientations, and these emotions were commonly observed among our participants. This distrust also encourages disengagement in social media. As such, the distrust associated with social media shifted the focus away from the assessment of the quality of information to the social media platforms themselves.

Interestingly, as shown in Figure 6, we see that those who were afraid of the potential harmful effects of misinformation (e.g., P09, P15, P16, P20) also had misinformation scores that were in the middle range, indicating that this group of participants believed that certain misinformation was likely to be "true". These participants were also more prone to believing misinformation than others in our study-those participants described in subsubsection 5.1 .2 (e.g., P02, P08, P11, P12). However, this group of people was aware of the limitations they experienced in distinguishing misinformation from "true" information.

Additionally, our findings indicate that social media distrust was built up in participants over time and was not necessarily a direct consequence of COVID-19. For example, P09 stated "I decided, many years ago to tune out of that", and P12 indicated "the social media thing [not engaging with social media] was preexisting”. This indicates that feelings of social media distrust are often linked to a multitude of prior experiences with misinformation, which we refer to as an individuals' historical past online.

Summary: Collectively, we found that issues of trust were the main driver for the diminished use of social media for COVID-19 information. It was mainly feelings of inability to change others' misbeliefs and mistrust that led participants to stop using social media. Our participants experienced various tensions and conflicts with their social media contacts, arising from the discrepancy between perceptions and beliefs regarding what to trust and whom to trust. While such tensions on social media existed prior to the pandemic, COVID-19 intensified and reignited these online conflicts. Our younger participants frequently reported challenges as they attempted to shift and correct their parents' or relatives' misbeliefs and mistrust. However, as a result of societal norms and a desire to maintain healthy relationships with family, our participants compromised by engaging less with social media. As such, these findings shed light on the generational gap and relationship dynamics that play a role in online trust.

Moreover, we found that although our participants used social media for consuming COVID-19 information, most of them did not trust this information. The most commonly cited reason for social media distrust was the perception that social media is a mixture of truthful information and misinformation. These perceptions led to a sense of insecurity, fearfulness, and skepticism regarding social media among participants. Concerns regarding the potential negative impacts of misinformation led participants to feel "afraid". Consequently, we observed a shift in trustees from the information on social media to the social media platform(s) themselves. This shift indicates that addressing the perceived and objective credibility of information might not be sufficient for solving the fundamental issues of trust on social media. Instead, these findings shed light on the deep challenges around distrust in social media infrastructure that need to be addressed.

\subsection{Trust in COVID-19 Data: Information Production \& Representation Concerns}

Other than the distrust in social media, we found that participants also expressed their distrust in the more fundamental informationthe COVID-19 data (i.e., the number of cases/deaths). In this subsection, we seek to answer two questions based on our survey results: Qc. in what ways are our participants' distrust associated with their information exposure? and Qd. why has respondents' distrust in authorities decreased over time with regards to the sharing practices of COVID-19 data?

5.2.1 Processes of Information Production. Some participants were skeptical about the processes through which COVID-19 data and messages were assembled and constructed, referred to here as the production of information. For example, P06 rated the statement that the authorities intentionally reported inaccurate COVID-19 data as "extremely likely" (=5) across all waves. During the interview, P06 explained why he questioned the validity of the COVID-19 data being produced:

"I don't trust [the COVID-19 data] because not everybody died from coronavirus. My friend's dad had heart failure and died. They took the COVID-19 PCR test and it was negative... But at the end, there was one piece of paper [death certificate] saying it's COVID-19. He said my dad didn't die from COVID-19 but just a heart failure... People passed [away] with the COVID-19 negative... somebody's getting paid for putting COVID-19 on [death certificates], so that contributes to the inflation number... put COVID-19 [on the death certificate] they 
get so much money from the federal government now."

(P06, 35-44, Male, High school or less, Republican)

P06's reasoning processes suggest that he relied on the information he received from his personal network (e.g., friends and coworkers). This information serves as a baseline for him to evaluate the trustworthiness and validity of COVID-19 data. Only relying on information from personal networks runs the risk of over-generalizing individual cases. We should note that P06 relied on second-hand information learned from other people. Thus, this knowledge may stem from rumors or misinformation.

Similar to P06, other participants $(n=5)$ relied heavily on their personal experiences and environmental cues to guide their assessment of the accuracy and trustworthiness of COVID-19 information. For example, participants, like P14, felt as though COVID-19 reports did not align with their own perceptions. According to the survey result as shown in Table 3, P14 believed that the authorities intentionally reported inaccurate COVID-19 data and explained,

"You see all these numbers and things like that, and

I'm like all these people that I've been around and close in my life, and you know, knowing a lot of people that have NOT gotten COVID...” (P14, 25-34, Female, High school or less, Republican)

These participants indicate that they were skeptical of the production of COVID-19 information, which contributes to their distrust in authorities.

While most participants who held skepticism were not involved in the collection of COVID-19 data, some who articulated their trust and distrust in COVID-19 data collection had first-hand experiences dealing with this process. One participant who worked in the public health sector described his daily job:

"I made sure that birth [and] death records are recorded properly... I work with hospitals, funeral homes, doctors, city and town clerks, anybody that has a hand in the vital record creation process or amendment process... I go out and make sure that those things get corrected and educate whoever made the mistakes... Mostly these days [we're] fixing death records [because] data is critical during COVID, and we were constantly trying to make sure that they were recorded properly. I don't actually clean the data, but I make sure that their records are done properly so that data is accurate." (P08, 45-54, Male, Graduate Degree, Independent)

P08 believed that the COVID-19 data was trustworthy, and he trusted the authorities who provided the data. This is reflected within his survey data, as well (see Table 3). P08 rated the statement "authorities intentionally report inaccurate COVID-19 data" as "extremely unlikely" (=1) across all waves. Yet, one might argue that it is the nature of P08's occupation (a member of staff working in the public health sector) that influenced his survey responses. However, surprisingly, as an "insider" who was involved in the data pipeline, P08 also had doubts regarding the COVID-19 data, as he explained:

"And right now, because of our famous reimbursement program, we're seeing a lot of COVID being added to death records. The doctor has to be okay with it, but when it does appear on the death record... [they] will reimburse family... so we're getting a lot of COVID added to record, so I'm not $100 \%$ sure what that's going to do to national statistics, but I would imagine that the numbers are going to go up as a contributor. I don't know whether or not it's a lot of people had preexisting conditions. I don't believe that a lot of people were tested at the time of death. They may have exhibited signs similar to COVID when they passed away from cardiac arrhythmia or something like that. So me personally, not speaking on behalf of [my employer], I think that the numbers are going to be a little artificially higher because of these programs, but it is what it is." (P08, 45-54, Male, Graduate Degree, Independent)

P08's reasoning process, as well as his conclusions regarding trust and distrust, suggests that he believed that the COVID-19 data in general was truthful but had doubts in the data production procedures. While P06 and P08, noted similar beliefs and concerns about economically-driven fraudulent medical documentation, to the best of our knowledge, there is no credible evidence of such a trend. Instead, this viewpoint has been cited as a known conspiracy theory $[12,22]$. The fact that two participants with very different backgrounds and worldviews hold similar beliefs about the situation suggests that their distrust in authorities was prompted by the skepticism in the COVID-19 data production processes.

When examining participants' quotes alongside their survey results, we find that the justification for trust and distrust differs across participants. On the one hand, P06's results demonstrate his high distrust in authorities. Such distrust arises from skepticism in the validity of COVID-19 data production processes, fueled by his past exchanges with his personal networks (e.g., friends and coworkers). However, in P08's case, he highly trusted authorities thanks to his first-hand experience interacting with the COVID-19 data production process, but he had reason to be suspicious in other respects. As such, the trust exists but is tempered by distrust. Our observations on the coexistence of trust and distrust is sometimes referred to as "bounded trust" [47]. This coexistence was also observed among other participants $(n=4)$, as well. For example, P09 explained how her trust and distrust was coupled together:

"I think the data and the protocols that rolled out were the best that they could provide us, given that they were still trying to figure out things. And I'm sure they had a desire to get it right to the best of their ability, so I trust the process that we went through... though I do think there was some suppression of data, like maybe more control that was needed from a government standpoint because they didn't want, in my opinion, people to freak out." (P09, 35-44, Female, Graduate Degree, Democrat)

We also find it important to highlight the clear disparity in beliefs between participants on the adjustment of COVID-19 data.

Things become more interesting when examining how participants' trust and distrust formation relates to their information practices, as well as the information sources, on which they have been relying. Take, for example, the results of P06, P08, and P14. Our findings suggest that these participants possessed distinct methods of trust and distrust formation. Triangulated with our survey data, our results also suggest that the trends and patterns of their information practices are dissimilar. 
First of all, with regards to information exposure, P06 reported that he received minimal COVID-19 information throughout the entirety of the survey period, whereas $\mathrm{P} 08$ had high information exposure. We observe similar trends among all participants in Figure 5. P06's label is located at the top-left corner of Figure 5, indicating that he had received little information about COVID-19 and had high distrust in the authorities who produced COVID-19 data. P08's data point is located at the bottom-right corner of Figure 5, indicating that he had received a lot of information about COVID-19 and had low distrust in authorities. Although these two data points appear to be opposite extremes, more people in our study were similar to P06, P08, and P14 (as shown in Figure 5). Additionally, Figure 5 shows that even with the same information exposure, people greatly differed in other characteristics.

Secondly, as previously mentioned, P06 and P14 relied heavily on information from personal networks in their assessment of information. These two participants mentioned in the interview that they refrained from searching for COVID-19 information online. For example, P14 explained,

"I really didn't search [COVID-19 information] because I really try to refrain from searching. I never went out looking for for information and or any of that." (P14, 25-34, Female, High school or less, Republican).

Unlike P06 and P14, P08 sought out information from multiple sources, including the CDC, Dr. Fauci, the national and local government, a variety of media outlets, and personal networks, like family and friends (see Table 3). P08's interview data aligned with his survey data, as he indicated that he "currently look[s] at cases almost every day". Taken together, these findings provide us with a "profile" of our participants, showcasing how trust and distrust formation intersects with their information practices.

5.2.2 Representation of Data Uncertainty. Skepticism is not only derived from the process of information production but also arises in the presentation of that data to information consumers. One challenge that arises from the wide range of COVID-19 data sources is data discrepancy - when two or more comparable datasets do not match up. COVID-19 data is widely available on the internet and is mostly open-sourced but has been published by various different organizations, authorities, and digital volunteers [101]. The discrepancy between currently-available COVID-19 data published by multiple different information sources overwhelmed our participants and made it difficult for them to decide whom to trust. As P20 explained,

"Because it was so mixed reporting, every outlet was reporting on it, and the numbers were kind of different; the differences in the numbers were like well who's telling the truth." (P20, 35-44, Male, Associate Degree, Republican)

P20 was unsure who to trust regarding the publication of COVID-19 data, which is reflected in Figure 5. To P20, there was no sufficient evidence to support his trust in authorities.

In addition, the wide range of publicly available data sources also led to the diverse manifestations of COVID-19 data visualizations. These COVID-19 visualizations were produced by different authorities and organizations that utilized different data sources and visualization techniques to present the data. These discrepancies within various COVID-19 visualizations generated confusion among some of our participants. For example, P16's survey results indicate that she believed authorities were very likely to intentionally report inaccurate COVID-19 data (see Figure 5). During the interview, P16 explained how discrepancies within visualizations made her feel distrustful towards the authorities who supplied the COVID-19 data:

"If I read The New York Times, watch ABC news, watch $C N N$ and BBC, you'll get four different figures, so how can there be four different figures?! And then it never you know certain things weren't cited that well." (P16, 45-54, Female, Graduate Degree, Republican)

The nuanced representations raised concern among participants and led to perceptions of data inconsistency, which contributed to the overall distrust in authorities.

A few participants $(n=2)$ described their trouble understanding the minor inconsistencies in the reported COVID-19 data. For example, P10 explained,

"Most statistical information presented on the media is a little shaky if you actually have done like grad level statistics... like if they say 250 people died on Monday, then you know, on Wednesday you can't say the total is 170. How do you have less people two days later than you did on Monday? So I'm just used to it being slightly inaccurate at best. So I don't know if they intentionally misreported anything. I think it's probably a combination of a little ignorance about statistics [and] people just rushing to put out a number for the sensation of it or to back up a story." (P10, 35-44, Male, Graduate Degree, Democrat)

P10's quote demonstrates his skepticism in the media, as he believes that bias may have inherently influenced COVID-19 data. This is exhibited in his belief that certain information outlets might just "put out a number for the sensation of it or to back up a story".

Prior work has suggested that uneducated or low-educated individuals have difficulty understanding statistics and numbers [21, 48]. Yet, in our study, some of the highly-educated participants (e.g., those with graduate degrees) did not appear to realize that statistical anomalies and/or methodology changes in the data are common in times of crises. Our participants were not aware that COVID-19 data is provisional and subject to change. Therefore, the reason why COVID-19 visualizations may have shown 250 deaths, for example, but later updated to show 170 deaths for the same day might be due to the imprecise nature of recently published data, which is affected by additional reporting and/or issue fixing [13].

However, we argue that understanding the complexities around COVID-19 data is unlikely our participants' fault. Instead, authorities and organizations fail to highlight the uncertainty of data processing and information representation techniques, which ultimately sacrifices the clarity of the information. Altogether, this lack of transparency contributes to the manifestation of distrust in authorities among individuals.

Additionally, similar to $\mathrm{P} 10$, we encountered several cases $(\mathrm{n}=5)$ in which participants elaborated on their distrust of COVID-19 information, citing media biases as the main culprit for their wariness, fear, and worries. For example, P02 indicated that, 
"Like everything, [information sources are] pushing their own agenda, and if I'm only reading content that pushes my own agenda as well, how am I, as a consumer of content, not going to be biased?" (P02, 18-24, Non binary, Bachelor degree, Democrat)

The political positionality of an information source influences and potentially biases the understanding and views of the actors (i.e., information consumer). Of course, one could argue that no source could assume a completely "neutral" position. Yet, in the context of the COVID-19 public health crisis, our participants indicated their willingness to seek out information sources that objectively communicate COVID-19 data and/or information. For example, P18 told us she relied on both the CDC and a crowdsourced sociotechnical system (i.e., the COVID Tracking Project) for COVID-19 information. She also emphasized her concerns about the mainstream media, as she explained how her trust in these media sources decreased during the pandemic:

"I got most of my news from the CDC and the COVID Tracking project that was on Twitter. [The COVID Tracking project] really just posted like daily statistics and offer different links to the COVID data sources... You know, I don't really trust the mainstream media-there's kind of bias on both sides. Not that I watch Fox news or that I've ever watched it, but even CNN that whole time is so daunting. Even when they are reporting on how many cases, how many people died, it was a little overwhelming... I think the [COVID Tracking Project] Twitter account was less daunting in some ways... I could just click on it and see what I needed to see and then move on from it... Maybe, because people weren't talking about it, maybe I could just read it. And I don't know I just remember being in my own space clicking on it look okay... I just maybe a little appreciated the silence of reading about it, instead of hearing them say the same thing over again..." (P18, 25-34, Female, Graduate Degree, Democrat)

P18 avoided traditional mainstream media, as she was afraid that media biases inherently influenced the messages being communicated. She noted that different news outlets set dissimilar tones in the communication of COVID-19 related information. The feelings and emotions (e.g., "feel daunting") that arose when obtaining COVID-19 information from CNN indicate her perception on sensationalism, or exaggerated messages and stories that emphasize fear. Her heightened distrust was colored by her wariness and concerns over media bias, which she considers to be inherently entrenched in the foundation of mainstream media outlets.

Meanwhile, her acceptance and engagement with emerging crowdsourced information systems (e.g., the COVID Tracking Project) reflects her shifting interest in seeking information sources with "neutral" positionality. This COVID Tracking Project [80] was created by a group of digital volunteers with the goal to address the growing amount of poor quality COVID-19 data (e.g., missing data, latency, etc.). The data collected and aggregated by the COVID-19 Project was utilized by two presidential administrations and an array of federal agencies, including the CDC and the FDA, over the course of the pandemic. Some participants believed that these crowdsourced sociotechnical systems were relatively unbiased in comparison to traditional mainstream media, which improved the trust levels of these sociotechnical sources. Cognizant of the media bias and polarization of media coverage during COVID-19, some participants shifted to information platforms with less editorial components.

To better understand the relationship between individuals' information practices and their distrust, we triangulated our survey data with our interviews. Several participants (i.e., P16, P20) who had relatively high information exposure (average scores $\geq 3.0$, indicating they obtained COVID-19 information at least 2-3 times per week) also tended to distrust authorities (average scores $>4.0$ ). It is worth noting that the discrepancies found in the data and visualizations indicate that these participants were seeking out and paying close attention to COVID-19 data. However, our findings suggest that exposure is necessary, but not sufficient, in building their trust.

5.2.3 Distrust in Authorities Decreases as Data Discrepancy Reduces. During our interviews, we explored why the distrust in authorities diminished among respondents over the course of our survey period (to answer Qd). Some participants felt as though the discrepancies among different COVID-19 sources reduced over time, which ultimately improved the trust in authorities among these participants. For example, P20 explained,

"When I took the survey earlier, [CNN] were putting like $X$ amount of numbers [e.g., cases], but then you turn to a nearby channel that says Georgia has a different amount of number... Now their reporting seems to be closer from outlet to outlet, so now I tend to trust it..." (P20, 35-44, Male, Associate Degree, Republican)

P20 indicated that over time, he built up trust in authorities because the COVID-19 data discrepancies across various outlets had (partially) reduced over time. Yet, P20 was unable to specify the root cause of the COVID-19 information convergence seen across different sources.

Here, we provide plausible explanations. At the beginning of the pandemic, many different information platforms were quickly created to support collecting, aggregating, and presenting COVID-19 data to the public. These different organizations were collecting and reporting COVID-19 data on their own, including multiple emerging crowdsourced systems (e.g., the COVID Tracking Project [80], the COVID Tracker by 1Point3Acres [1]) and news outlets (e.g., the New York Times [81]). At that time, there was a shortage of centralized, reliable data sources, forcing even the CDC to utilize data from these crowdsourced websites. Yet, over the course of the pandemic, the centralized information platforms, namely those supported by the federal agencies, improved. These federal agencies (i.e., the $\mathrm{CDC}$ ) were able to provide high-quality data to many different information outlets, which many sources referred to on their websites as "raw" data. This is reflected in the COVID Tracking Project's farewell statement on their website, explaining that "as we wind down at the one-year point, we are both satisfied that the federal government is now producing enough data to replace many of our metrics and eager to turn our attention to the analysis of those areas of the data-like race and ethnicity data" [80]. Over time, COVID-19 data production and presentation have become more stabilized and 
centralized. These perceptions of stabilization across COVID-19 information help us understand how trust has emerged and developed among our participants (i.e., P20, P16, P8, P19).

Summary: By triangulating our survey results and interview data, we found that skepticism among participants originated in both the data production and data representation processes. With regards to data production, we conclude that people's lack of exposure to information, as well as their reliance on personal networks, may correlate with the inability to identify misinformation. In contrast, past experience in evaluating information can influence one's ability and willingness to establish and balance trust and distrust. Such an ability may enhance people's understanding of the limitations in COVID-19 information, ultimately helping them navigate the chronic uncertainty amidst the pandemic. With regards to data representation, the discrepancies between information sources, as well as the inadequate transparency and clarity regarding data uncertainty, raised concerns and skepticism among participants. Understanding these issues helped us identify the mechanisms for which distrust manifests, highlighting the importance of identifying design constraints in sociotechnical systems and information infrastructure.

\section{DISCUSSION}

Our survey results and interview findings collectively characterize a variety of factors that contribute to trust and distrust formation, taking into account people's information practices. In this section, building upon our findings, we reflect on the broader challenges of existing information infrastructure that have contributed to the trust and distrust formation. We also provide implications for future work to examine trust and information behaviors.

\subsection{Deep Challenges in Information Infrastructure}

Below we discuss broader issues of the existing information infrastructures that have shaped play critical roles in trust and distrust formation amidst COVID-19, and implications for future work.

6.1.1 Trusting \& Distrusting While Infrastructuring. Throughout the pandemic, COVID-19 data has been made available through both well-established social media platforms, as well as newlyestablished information platforms, such as crowdsourced systems. These information platforms have integrated into the information infrastructures for crisis response. By definition, information infrastructure refers to networks of heterogeneous sociotechnical systems [46] ${ }^{12}$. In times of crises, a collection of information infrastructures are created and developed-sometimes also referred to as "crisis infrastructure" [19]. These platforms together constitute the multi-infrastructural contexts [19] in which crisis and risk information gets distributed to the public.

Our work examined how distrust manifests among our participants, both in terms of participants' concern, as well as their skepticism, regarding COVID-19 data production and data representation.

\footnotetext{
${ }^{12}$ Information infrastructure has a wide range of meanings $[6,35,46]$ and can refer to different concepts, such as information itself, applications and software, the network stands and transmission codes, or simply the people who create the information system. A more recent practice, and one we adopt in this paper, is using information infrastructure to refer to networks of sociotechnical systems.
}

We argue that a fundamental reason for their distrust lies in the "failure" of existing information infrastructures. During the pandemic, reporting and presenting COVID-19 data is challenging because of the lack of comprehensive guidelines for COVID-19 data collection [71]. Our participants were not even sure who held the "official" data. For example, in the United States, the CDC was slow to deliver COVID-19 data at the beginning of the pandemic and needed to rely on crowdsourced systems [71, 80, 83]. Each state's Department of Public Health, news media outlets, and crowdsourced organizations all had their own data practices and pipelines and differed in their data collection, data compiling and aggregation methods, as well as their visualization, and reporting techniques. Thus, the COVID-19 data and its representation were inconsistent across information outlets $[71,101]$. The failure to establish proper information infrastructure in response to the crisis led to issues of trust among our participants. COVID-19 data from these platforms often serve as "facts" that reflect the near-real-time pandemic situation. As such, this data is used to guide policy-making, facilitate sensemaking around the crisis, and help the people stay informed. Likewise, it informs their risk perception and guides their behavior to mitigate risks. However, when people start questioning the validity and accuracy of the COVID-19 data, they begin to lose trust and faith in the authorities who collect and report the essential data. Consequently, this distrust may further impact their perceived severity of the crisis, influencing their decision-making and behaviors.

Meanwhile, our results also show that participants' distrust decreased over time. This decrease of distrust is partially due to the gradual way in which information infrastructures were established and improved in response to COVID-19. We can further understand the evolution of trust amidst the pandemic by engaging with the concept of infrastructuring. "Infrastructuring", which originated in work by Star and Ruhleder [74] and was coined by Pipek and Wulf [62], emphasizes the processes and practices involved in the evolution of infrastructures. Infrastructuring in the context of the COVID-19 pandemic refers to the ways in which we are constantly revising our feelings of "trust" and "distrust" as new information infrastructures evolve. Therefore, our work demonstrates how trust and distrust evolves alongside the changes in the information infrastructure in times of crisis. By unpacking various issues of trust with regards to information platforms amidst COVID-19, our work contributes to an in-depth examination of the ways in which social media, as well as the emerging COVID-19 data platforms, enable and constrain public trust.

6.1.2 The Role of Social Media in Crisis Infrastructure. In addition, social media plays an important, yet controversial, role in crisis infrastructure. Social media can facilitate the exploration and communication of risk information during crises [4]. However, the spread of misinformation on social media is also problematic [75]. Recent work shows that the proliferation of misinformation on social media hinders and distorts individuals' information consumption during crises like COVID-19 [60, 78] and suggests that people's reliance on social networks has been remarkably high throughout the pandemic. Our findings refute this aforementioned work, given that many of our participants "used" social media for COVID-19 information consumption, but many did not necessarily "rely on" nor "trust" this information. Some of our participants felt 
as though they could distinguish between misinformation and "true" information, and our survey findings showed that they were indeed less likely to believe in the misinformation on social media. Other people in our study decided to avoid social media for COVID-19 information due to insecurity, fear, and skepticism regarding the harmful effects of misinformation on social media, as well as their uncertainty in their abilities to discern misinformation. In subsection 6.2, we further reflect on the discrepancies identified between our findings and other work that has previously examined trust in relation to information practices.

Moreover, some decided to stop using social media platforms altogether for any and all information, leading to the non-use of social media. The topic of non-use amidst sociotechnical systems, such as social media, has gained increasing attention in HCI literature [31, 68]. Built upon Satchell and Dourish's non-use conceptual framework [68], recent work by Grandhi et al. [31] specifically examined the different rationale for non-use on social media, such as active resistance, disenchantment, disenfranchisement, disinterest, replacement, and cognitive bias, all of which can lead to information avoidance. Our findings add new insight into this emerging body of work, specifically demonstrating the relationship between non-use and trust, as well as relating non-use to the unique challenges of sub-populations (e.g., low-SES groups) within our research focus .

Amidst the pandemic, social media ignites tension and conflicts between people, causing anxiety and stress on relationships among many users according to our participants. This discord between individuals' preexisting beliefs and the beliefs of others on social media generates a strong enough sense of "incapability" and "powerlessness" among users to persuade them to adopt information avoidance behaviors as a response. Prior work has shown that people engage with social media to maintain personal and social relationships [31], yet our findings suggest that people actually avoid social media as a way to maintain healthy relationships. This directly contradicts prior research on the relationship between social media non-use and social relationships, as reduced social media use has been shown to be positively correlated with a lower connectedness with others [58]. Given the stress that is induced by feelings of loneliness, it is likely that people revert back to social media platforms that they previously quit [31], as prior work suggested. As such, these findings collectively indicate that the use and non-use of social media platforms change over time and depend on specific contexts. More research is needed to further examine the complex relationship between trust, use and non-use, and physiological well-being.

Moreover, it is important to point out that many topics unrelated to COVID-19 have and always will cause conflict among individuals given the multitude of diverse perspectives on social media. However, the pandemic, by its very nature, has caused these conflicts among users to greatly intensify, generating extreme contention and high levels of frustration and compelling many to cease their social media use. Individual factors, such as the age differences [33], social norms [34], and political affiliation [34], have been shown to influence the belief and dissemination of misinformation. These findings directly coincided with our interview findings, as participants often cited tensions they experienced with older individuals in their lives who had opposing views, coupled with an attempt to avoid conflict and comply with social norms, as a major driver in their information avoidance behaviors on social media.

Further, the examination of non-use in our study is situated in the context of the studied populations (e.g., lower-SES communities). Prior work has shown that active resistance to social media can stem from a lack of time for the general population [31, 68]. Our work suggests that additional characteristics help explain the non-use in our focused population. Our interviews uncovered that our low-SES participants lacked the time and energy to engage with social media during the reopening phase of 2020, as many of them worked in "essential services", forcing them to attend work physically. Additionally, prior work has not sufficiently unpacked the effects of the non-use. While active resistance to using social media may have distinct positive outcomes for users, such as an increase in time for physical activity [31], our findings suggest that this same active resistance to social media platforms contributes to a reduced consumption of social media information. Our participants further described how their non-use of social media for COVID-19 information consumption was also driven by their collapsing trust in social media that occurred prior to the pandemic. For individuals with this kind of historical distrust in social media, it might be extremely difficult to rebuild their trust system in social media platforms.

It is important to note that social media infrastructure is also evolving. For example, social media platforms have improved the detection of misinformation, improving the overall quality of online information [3, 70, 97]. However, simply targeting the quality of information is not sufficient in solving the issues of trust within social media. Our findings shed light on fundamental issues surrounding trust, taking into account the challenges of trust revision (i.e., changing other's mistrust and misbelief), tensions and conflicts between generations, and differences among particular subpopulations. Our findings suggest that simple interventions, such as using AI to remove misinformation on social media, may not be sufficient. Instead, we highlight the necessity to shift the focus to designing for trust revision and tension mitigation as a long-term goal of establishing and reserving trust. Such explorations are especially critical during crises where many conflicting values need to be assessed by the information consumers in a short amount of time. It is difficult, if not impossible, to find a simple solution for such a complex problem in the midst of a crisis, and thus, more research is needed to explore interventions that aid in both trust revision and tension mitigation to improve the crisis infrastructure.

6.1.3 Implications for Future Work. Our work examined how people's trust and distrust emerges, develops, and collapses given various types of trustees, such as authorities and information sources who report and present COVID-19 data, as well as social media in general. In fact, every information consumer is impacted by all these information sources and information platforms. The sources and platforms, together with the consumers, form a complex information flow and trust/distrust network.

We encourage more research to consider the joint impact that stakeholders have in both the information and trust/distrust networks, paying close attention to the collaborative effort required to build infrastructures under time pressures. These stakeholders hold 
their own strengths and limitations and can thus benefit from learning from one another. For example, the New York Times COVID coverage was awarded the 2021 Pulitzer Prize for Public Service [82]. Likewise, the COVID Tracking Project has recently been archived as an open-source data infrastructure by research institutions [87] To the best of our knowledge, this was the first time in the digital age that the documentation of crowdsourcing activities became a part of the key infrastructure in the country. Explicitly examining the collaboration across organizations and authorities will not only contribute to crisis informatics, $\mathrm{HCI}$, and public health research but will also help document human evolution and provide a new way of preserving our history. As we start documenting and sharing lessons learned from both information providers and consumers, addressing the institutional challenges and adjusting the information infrastructure, we hope to help the general public improve their trust systems and better prepare for future crises.

Moreover, our findings suggest that feelings of distrust among participants were deeply shaped by experiences they had prior to the pandemic. Our findings also indicate that a shift in trustee (i.e., who and what is trusted) occurred among participants, fluctuating between distrust in the information on social media and distrust in the social media platforms themselves. Temporality plays an essential role in this shift. Participants' accumulated negative experiences with misinformation are reflected in their perception of social media platforms as a whole. In other words, small, temporal units of engagement and negative experiences with misinformation in one's past can lead to distrust in the social media platform. It is important that we seriously consider the insights gathered throughout the COVID-19 crisis to improve the crisis infrastructure and foster trust. Otherwise, as we have highlighted in this paper, trust is easily broken but difficult to regain.

\subsection{Methodological Reflections}

Drawing upon our experiences in this research project, we conclude with reflections on methodological opportunities and challenges that are important for research on trust and information behaviors. In this section, we discuss previous work focused on the association between trust and information practices to highlight the importance of mixed-methods research when assessing these relationships.

Our triangulated data sheds light on an important research gap regarding the relationship between trust and information consumption. A vast majority of previous work has explored the relationship between trust and information practices by assessing individuals' use of information sources to obtain COVID-19 information $[28,50,56,60,96,98]$. For example, prior research has explored the relationship between perceived trust and information sources by first identifying if individuals "use" (e.g., [98]) or "follow" (e.g., [56]) a particular source to obtain COVID-19 information. However, we argue that great care must be taken in what is inferred from the answers to those questions. For example, while prior work has drawn conclusions about what information sources people "rely on" based on where they have gotten information from $[45,84,96]$, our results paint a different picture. Specifically, we found that despite reporting that they get COVID-19 information on social media, many of our interviewees did not trust this information and did not rely on it to make decisions. This raises questions about what it means to "rely" on information (e.g., simply assessing reliance as the extent to which information is gathered from a source versus how much it is used to make decisions). Such concepts must be clearly defined and measured to support accurate and robust knowledge building around these phenomena. Furthermore, our nuanced understanding of participants' use of and trust in information was surfaced only as we triangulated our survey and interview data, with our interviews allowing us to probe the trends arising in our survey. As such, we make two recommendations. First, mixed method studies are essential for truly unpacking the dynamics of information trust and distrust formation. Second, given the different measurements and word choices used in current COVID-19 survey studies, we believe a meta-review that characterizes the varied approaches to assessing information practices, reliance, and trust would be beneficial for future research.

Additionally, prior survey studies measuring perceived trust in information sources $[28,50,60]$ have not specified the particular information that respondents relied on to make eventual decisions. Furthermore, individuals' levels of trust can change over time. Therefore, one-time assessments of trust in relation to people's information practices fail to accurately capture the dynamics of trust and distrust formation. Even longitudinal survey studies have limitations, as we cannot fully capture peoples' changing perceptions and shifting evaluations of trust. Therefore, we encourage future research to consider combining longitudinal survey analysis with repeated interviewing methods [57], incorporating recurring interviews among the same research population to better understand how, when, and under what conditions their trust and distrust start to change. Although more time-consuming, leveraging the longitudinal survey with recurrent interviews would facilitate a deeper understanding of the capricious nature of trust.

Moreover, our results showed that low-SES groups in our study had significantly higher trust in their personal contacts and significantly lower trust in Dr. Fauci. Furthermore, they utilized the internet and social media significantly less to gain COVID-19 information. Additionally, in our interviews, some participants from low-SES households described how their communities had suffered from mistrust issues. Their inability to change the misbeliefs and mistrust of the people surrounding them deeply disappointed our participants. Arguably, these issues may also occur within other populations, such as higher-SES groups. However, our work seeks to avoid the assumption that all populations have similar concerns, perspectives, and processes of trust and distrust formation. Such assumptions can be harmful to marginalized populations, generating systems that disproportionately benefit more advantaged groups $[94,100]$. Further research is needed to investigate the prevalence and reasons behind these trends across varied demographic groups, as well as in larger sample sizes. Such research will need to overcome potential barriers to engaging marginalized populations in research studies. For example, while a concerted effort was made to reach out to all of our survey respondents who expressed interest in participating in a follow-up interview and to recruit a diverse sample of interviewees, we had higher response rates from respondents with higher education and income levels. In turn, we were constrained in our attempts to study the experiences of varied demographic groups. Therefore, we strongly encourage additional research that examines a broader range of marginalized 
groups to better understand their strengths, concerns, skepticism, and difficulties regarding their trust and distrust formation.

\section{LIMITATIONS}

In our survey study, as mentioned in the method, we examine only a small set of information behaviors during COVID-19 in this paper. Relatedly, due to space considerations, we did not present the survey results and interview findings regarding the information practice and trust differences between MA and GA respondents. We will report the geographical differences in follow-up reports. Moreover, our survey respondents were asked to answer their information consumption "in the past 2 weeks", which may be limited by the fallible accuracy of individuals' past recollection. Additionally, we focused on the perceptions and trust people had towards social media platforms and emerging COVID-19 data platforms in general rather than any specific platform. As our findings shed light on the general issues of information infrastructure, we believe there is a need to narrow down the scope by specifically examining issues of trust within each individual platform. In our interview study, we had a greater number of participants who received higher education levels (see Table 1). These sample limitations restrict the generalizability of our findings to the broader U.S. population. Still, our findings provide important insight into the information practices and attitudes of our participants and highlight several important areas for future work to investigate.

\section{CONCLUSION}

The study of the dynamics of trust is critical for handling current and future crises. Our findings characterize 1) the ways in which the trustee (i.e., what/who people trusted) shifts from information from social media to the social media platform(s); 2) how distrust in authorities regarding COVID-19 data shifts over time; and 3) how the trust shifts from traditional news media to crowdsourced platforms. Trust shapes, and is shaped by, the technologies and information infrastructures in complex ways. As information providers and authorities navigate through the COVID-19 crisis and continue to improve the systems and infrastructure, we may continue to observe changes in public trust. Meanwhile, as people continue interacting with various systems that enable information exchange, we may also expect their concerns, skepticism, and distrust to redefine the current design of sociotechnical systems and information infrastructures. As such, people are practicing their "trusting" and "distrusting" alongside the processes of "infrastructuring". How trust emerges, develops, and collapses relies on concrete conditions in which people utilize to seek out information within the information platforms. It is these concrete situations that determine which sociotechnical systems take root and thrive and which wither and die. The complexities that arise in the formation and evolvement of trust and distrust, alongside the relationship with people's information practices, will continue to be a challenging topic; one of which deserves more research in the future.

\section{ACKNOWLEDGMENTS}

This material is based on work that is partially funded by an unrestricted gift from Google. This research was also supported by
Northeastern University. We thank our participants for their support within our research. We also thank Niharika Mathur for helping with the interview data collection, the Wellness Technology lab at Georgia Tech for feedback, as well as our anonymous reviewers for their reviews.

\section{REFERENCES}

[1] 1Point3Acres. 2021. Global COVID-19 Tracker \& Interactive Charts. Retrieved 2021-08-12 from https://coronavirus.1point3acres.com

[2] Elissa M Abrams and Stanley J Szefler. 2020. COVID-19 and the impact of social determinants of health. The Lancet Respiratory Medicine 8, 7 (2020), 659-661.

[3] Mabrook S Al-Rakhami and Atif M Al-Amri. 2020. Lies kill, facts save: detecting COVID-19 misinformation in twitter. Ieee Access 8 (2020), 155961-155970. https://doi.org/10.1109/ACCESS.2020.3019600

[4] Melissa Bica, Julie L. Demuth, James E. Dykes, and Leysia Palen. 2019. Communicating Hurricane Risks: Multi-Method Examination of Risk Imagery Diffusion. Association for Computing Machinery, New York, NY, USA, 1-13. https://doi.org/10.1145/3290605.3300545

[5] Melissa Block. 2020. Meet The Team Behind The Coronavirus Tracker Watched By Millions. Retrieved 2021-09-06 from https://www.npr.org/2020/04/13/833073670/mapping-covid-19-millionsrely-on-online-tracker-of-cases-worldwide

[6] Christine L. Borgman. 2007. Scholarship in the Digital Age: Information, Infrastructure, and the Internet. The MIT Press, Cambridge, Mass. https: //doi.org/10.7551/mitpress/7434.001.0001

[7] Nadia M Brashier and Daniel L Schacter. 2020. Aging in an era of fake news. Current directions in psychological science 29, 3 (2020), 316-323. https://doi.org/ 10.1177/0963721420915872

[8] Cati G Brown-Johnson, Lindsay M Boeckman, Ashley H White, Andrea D Burbank, Sjonna Paulson, and Laura A Beebe. 2018. Trust in health information sources: survey analysis of variation by sociodemographic and tobacco use status in Oklahoma. FMIR public health and surveillance 4, 1 (2018), e6260. https://doi.org/10.2196/publichealth.6260

[9] Deborah Bunker. 2020. Who do you trust? The digital destruction of shared situational awareness and the COVID-19 infodemic. International fournal of Information Management 55 (2020), 102201. https://doi.org/10.1016/j.ijinfomgt. 2020.102201

[10] C Shawn Burke, Dana E Sims, Elizabeth H Lazzara, and Eduardo Salas. 2007. Trust in leadership: A multi-level review and integration. The leadership quarterly 18, 6 (2007), 606-632. https://doi.org/10.1016/j.leaqua.2007.09.006

[11] Celeste Campos-Castillo and Denise Anthony. 2019. Situated trust in a physician: Patient health characteristics and trust in physician confidentiality. The Sociological Quarterly 60, 4 (2019), 559-582. https://doi.org/10.1080/00380253. 2018.1547174

[12] CBS19. 2021. VERIFY: Are doctors inaccurately reporting deaths as COVID-19related? Retrieved 2021-09-06 from https://www.cbs19.tv/article/news/health/ verify-covid-19-reporting-and-death-certificates/501-cd4f1995-e342-4451ae90-a814f1ace 284

[13] Centers for Disease Control and Prevention. 2021. About CDC COVID-19 Case and Death Data. Retrieved 2021-09-06 from https://www.cdc.gov/coronavirus/ 2019-ncov/covid-data/about-us-cases-deaths.html

[14] Centers for Disease Control and Prevention. 2021. COVID-19 Data Tracker. Retrieved 2021-09-06 from https://covid.cdc.gov/covid-data-tracker

[15] Apoorva Chauhan and Amanda Lee Hughes. 2020. Trustworthiness Perceptions of Social Media Resources Named after a Crisis Event. Proceedings of the ACM on Human-Computer Interaction 4, CSCW1 (2020), 1-23. https://doi.org/10.1145/ 3392849

[16] John Child. 2001. Trust-the fundamental bond in global collaboration. Organizational dynamics 29, 4 (2001), 274-288.

[17] CNN. 2021. Tracking Covid-19 cases in the US. Retrieved 2021-09-05 from https: //www.cnn.com/interactive/2020/health/coronavirus-us-maps-and-cases

[18] Steven C Currall and Timothy A Judge. 1995. Measuring trust between organizational boundary role persons. Organizational behavior and Human Decision processes 64, 2 (1995), 151-170. https://doi.org/10.1006/obhd.1995.1097

[19] Dharma Dailey and Kate Starbird. 2017. Social media seamsters: Stitching platforms \& audiences into local crisis infrastructure. In Proceedings of the 2017 ACM Conference on Computer Supported Cooperative Work and Social Computing (Portland, Oregon, USA) (CSCW '17). Association for Computing Machinery, New York, NY, USA, 1277-1289. https://doi.org/10.1145/2998181.2998290

[20] Daniel Devine, Jennifer Gaskell, Will Jennings, and Gerry Stoker. 2021. Trust and the Coronavirus Pandemic: What are the Consequences of and for Trust? An Early Review of the Literature. Political Studies Review 19, 2 (2021), 274-285.

[21] Darren A DeWalt, Nancy D Berkman, Stacey Sheridan, Kathleen N Lohr, and Michael P Pignone. 2004. Literacy and health outcomes. Fournal of general internal medicine 19, 12 (2004), 1228-1239.

[22] EJ Dickson. 2021. Anti-Vax Doctor Promotes Conspiracy Theory That Death Certificates Falsely Cite COVID-19. Retrieved 2021-09-06 
from https://www.rollingstone.com/culture/culture-features/anti-vax-doctorcovid-19-death-certificates-984407

[23] Tristan Endsley, Yu Wu, and James Reep. 2014. The source of the story: Evaluating the credibility of crisis information sources. , 160-164 pages. 11th International Conference on Information Systems for Crisis Response and Management, ISCRAM 2014 ; Conference date: 01-05-2014 Through 01-05-2014.

[24] Ian Falk and John Guenther. 1999. Role of situated trust in rural sustainability: "levels of trust" community profile. https://doi.org/10.5465/amr.1998.926617

[25] Maria J. Figueiras, Jihane Ghorayeb, Mariana V. C. Coutinho, JoÃ £o MarÃŽco, and Justin Thomas. 2021. Levels of Trust in Information Sources as a Predictor of Protective Health Behaviors During COVID-19 Pandemic: A UAE CrossSectional Study. Frontiers in Psychology 12 (2021), 2780. https://doi.org/10.3389/ fpsyg.2021.633550

[26] Garrett M Fitzmaurice and Caitlin Ravichandran. 2008. A primer in longitudinal data analysis. Circulation 118, 19 (2008), 2005-2010.

[27] Andrea Forte, Nazanin Andalibi, Thomas Park, and Heather Willever-Farr. 2014 Designing Information Savvy Societies: An Introduction to Assessability. In Proceedings of the SIGCHI Conference on Human Factors in Computing Systems (Toronto, Ontario, Canada) (CHI '14). Association for Computing Machinery, New York, NY, USA, 2471-2480. https://doi.org/10.1145/2556288.2557072

[28] Ilona Fridman, Nicole Lucas, Debra Henke, and Christina K Zigler. 2020. Association between public knowledge about COVID-19, trust in information sources, and adherence to social distancing: cross-sectional survey. FMIR public health and surveillance 6, 3 (2020), e22060. https://doi.org/10.2196/22060

[29] Christine Geeng, Savanna Yee, and Franziska Roesner. 2020. Fake News on Facebook and Twitter: Investigating How People (Don't) Investigate. In Proceedings of the $2020 \mathrm{CHI}$ Conference on Human Factors in Computing Systems (Honolulu, HI, USA) (CHI '20). Association for Computing Machinery, New York, NY, USA 1-14. https://doi.org/10.1145/3313831.3376784

[30] Ted Goertzel. 1994. Belief in Conspiracy Theories. Political Psychology 15, 4 (1994), 731-742. http://www.jstor.org/stable/3791630

[31] Sukeshini A. Grandhi, Linda Plotnick, and Starr Roxanne Hiltz. 2019. Do I Stay or Do I Go? Motivations and Decision Making in Social Media Non-Use and Reversion. Proc. ACM Hum.-Comput. Interact. 3, GROUP, Article 235 (Dec. 2019), 27 pages. https://doi.org/10.1145/3361116

[32] Joost Oude Groeniger, Kjell Noordzij, Jeroen van der Waal, and Willem de Koster 2021. Dutch COVID-19 lockdown measures increased trust in government and trust in science: A difference-in-differences analysis. Social Science \& Medicine 275 (2021), 113819. https://doi.org/10.1016/j.socscimed.2021.113819

[33] Andrew Guess, Jonathan Nagler, and Joshua Tucker. 2019. Less than you think: Prevalence and predictors of fake news dissemination on Facebook. Science advances 5, 1 (2019), eaau4586. https://doi.org/10.1126/sciadv.abf1234

[34] Daniel Halpern, Sebastián Valenzuela, James Katz, and Juan Pablo Miranda 2019. From Belief in Conspiracy Theories to Trust in Others: Which Factors Influence Exposure, Believing and Sharing Fake News. In Social Computing and Social Media. Design, Human Behavior and Analytics, Gabriele Meiselwitz (Ed.) Springer International Publishing, Cham, 217-232.

[35] Ole Hanseth and Eric Monteiro. 1998. Understanding information infrastructure.

[36] Donna Haraway. 1988. Situated knowledges: The science question in feminism and the privilege of partial perspective. Feminist studies 14, 3 (1988), 575-599.

[37] Richard Heeks. 1999. Information and communication technologies, poverty and development.

[38] Tamanna Hossain, Robert L Logan IV, Arjuna Ugarte, Yoshitomo Matsubara, Sean Young, and Sameer Singh. 2020. COVIDLies: Detecting COVID-19 misinformation on social media.

[39] Will Jennings. 2020. Covid-19 and the 'Rally-Round-the Flag'Effect, UK in a Changing Europe. Luettu 1 (2020), 2020.

[40] Johns Hopkins University. 2021. COVID-19 Data Repository by the Center for Systems Science and Engineering (CSSE) at Johns Hopkins University. Retrieved 2021-08-19 from https://github.com/CSSEGISandData/COVID-19

[41] Audrey Kobayashi. 2019. International encyclopedia of human geography. Elsevier, Amsterdam, The Netherlands.

[42] John Koetsier. 2020. Reddit, Facebook, Twitter Worst For Mental Health Post-Coronavirus; YouTube Best. Retrieved 2021-11-19 from https://www.forbes.com/sites/johnkoetsier/2020/04/26/reddit-worst-formental-health-for-covid-19-news-consumption-survey-says

[43] Yubo Kou, Xinning Gui, Yunan Chen, and Kathleen Pine. 2017. Conspiracy Talk on Social Media: Collective Sensemaking during a Public Health Crisis. Proc. ACM Hum.-Comput. Interact. 1, CSCW, Article 61 (dec 2017), 21 pages. https://doi.org/10.1145/3134696

[44] Roberta Lamb, Steve Sawyer, and Rob Kling. 2000. A social informatics perspective on socio-technical networks.

[45] David Lazer, Jon Green, Katherine Ognyanova, Matthew Baum, Jennifer Lin, James Druckman, Roy H Perlis, Mauricio Santillana, and Ata Uslu. 2021. The COVID States Project\# 57: Social media news consumption and COVID-19 vaccination rates.
[46] Charlotte P Lee and Kjeld Schmidt. 2018. A bridge too far?: Critical remarks on the concept of "infrastructure" in computer-supported cooperative work and information systems. https://doi.org/10.1093/oso/9780198733249.003.0006

[47] Roy J Lewicki, Daniel J McAllister, and Robert J Bies. 1998. Trust and distrust: New relationships and realities. Academy of management Review 23, 3 (1998), 438-458. https://doi.org/10.5465/amr.1998.926620

[48] Isaac M Lipkus. 2007. Numeric, verbal, and visual formats of conveying health risks: suggested best practices and future recommendations. Medical decision making 27, 5 (2007), 696-713. https://doi.org/10.1177/0272989X07307271

[49] Alessandro Lovari. 2020. Spreading (dis) trust: Covid-19 misinformation and government intervention in Italy. Media and Communication 8, 2 (2020), 458461.

[50] Linqi Lu, Jiawei Liu, Y Connie Yuan, Kelli S Burns, Enze Lu, and Dongxiao Li. 2021. Source Trust and COVID-19 Information Sharing: The Mediating Roles of Emotions and Beliefs About Sharing. Health Education \& Behavior 48, 2 (2021), 132-139. https://doi.org/10.1177/1090198120984760

[51] Rachel Lutz. 2020. Gathering COVID-19 Data More Important Than Ever. Retrieved 2021-09-06 from https://www.contagionlive.com/view/gatheringcovid19-data-more-important-than-ever

[52] Apoorva Mandavillit. 2021. The U.S. Is Getting a Crash Course in Scientific Uncertainty. Retrieved 2021-09-06 from https://www.nytimes.com/2021/08/22/ health/coronavirus-covid-usa.html

[53] Stephen Marsh and Mark R Dibben. 2005. Trust, untrust, distrust and mistrustan exploration of the dark (er) side. In International conference on trust management. Springer, Springer Berlin Heidelberg, Berlin, Heidelberg, 17-33. https: //doi.org/10.1007/11429760_2

[54] Roger C Mayer, James H Davis, and F David Schoorman. 1995. An integrative model of organizational trust. Academy of management review 20, 3 (1995), 709-734.

[55] D Harrison McKnight, Larry L Cummings, and Norman L Chervany. 1998. Initial trust formation in new organizational relationships. Academy of Management review 23, 3 (1998), 473-490. https://doi.org/10.5465/amr.1998.926622

[56] Jad Melki, Hani Tamim, Dima Hadid, Maha Makki, Jana El Amine, and Eveline Hitti. 2021. Mitigating infodemics: The relationship between news exposure and trust and belief in COVID-19 fake news and social media spreading. Plos one 16, 6 (2021), e0252830. https://doi.org/10.1371/journal.pone.0252830

[57] Robert King Merton. 1968. Social theory and social structure (1968 enl. ed. ed.). Free Press, New York.

[58] Kaloyan Mitev, Netta Weinstein, Sonya Karabeliova, Thuy-vy Nguyen, Wilbert Law, and Andrew Przybylski. 2021. Social Media Use Only Helps, and Does Not Harm, Daily Interactions and Well-Being. https://doi.org/10.1037/tmb0000033 https://tmb.apaopen.org/pub/social-media-abstinence-and-interactions.

[59] National Governors Association. 2021. Coronavirus State Actions. Retrieved 2021-08-19 from https://www.nga.org/coronavirus-state-actions-all

[60] Stephen Neely, Christina Eldredge, Ron Sanders, et al. 2021. Health Information Seeking Behaviors on Social Media During the COVID-19 Pandemic Among American Social Networking Site Users: Survey Study. Fournal of Medical Internet Research 23, 6 (2021), e29802. https://doi.org/10.2196/29802

[61] Kathleen H Pine, Myeong Lee, Samantha A. Whitman, Yunan Chen, and Kathryn Henne. 2021. Making Sense of Risk Information amidst Uncertainty: Individuals' Perceived Risks Associated with the COVID-19 Pandemic. In Proceedings of the 2021 CHI Conference on Human Factors in Computing Systems. Association for Computing Machinery, New York, NY, USA, Article 653, 15 pages. https: //doi.org/10.1145/3411764.3445051

[62] Volkmar Pipek and Volker Wulf. 2009. Infrastructuring: Toward an integrated perspective on the design and use of information technology. Fournal of the Association for Information Systems 10, 5 (2009), 1. https://doi.org/10.17705/ 1jais.00195

[63] Qualtrics. 2021. Unlock breakthrough insights with market research panels. Retrieved 2021-08-12 from https://www.qualtrics.com/research-services/onlinesample

[64] Peter Railton. 2014. Reliance, trust, and belief. Inquiry 57, 1 (2014), 122-150.

[65] Christian Reuter, Amanda Lee Hughes, and Marc-André Kaufhold. 2018. Social media in crisis management: An evaluation and analysis of crisis informatics research. International Journal of Human-Computer Interaction 34, 4 (2018), 280-294. https://doi.org/10.1080/10447318.2018.1427832

[66] Maritza Vasquez Reyes. 2020. The disproportional impact of COVID-19 on African Americans. Health and Human Rights 22, 2 (2020), 299.

[67] Jon Roozenbeek, Claudia R Schneider, Sarah Dryhurst, John Kerr, Alexandra LJ Freeman, Gabriel Recchia, Anne Marthe Van Der Bles, and Sander Van Der Linden. 2020. Susceptibility to misinformation about COVID-19 around the world. Royal Society open science 7, 10 (2020), 201199. https: //doi.org/10.1098/rsos.201199

[68] Christine Satchell and Paul Dourish. 2009. Beyond the User: Use and Non-Use in HCI. In Proceedings of the 21st Annual Conference of the Australian ComputerHuman Interaction Special Interest Group: Design: Open 24/7 (Melbourne, Australia) (OZCHI '09). Association for Computing Machinery, New York, NY, USA, 9-16. https://doi.org/10.1145/1738826.1738829 
[69] Jae M Sevelius, Luis Gutierrez-Mock, Sophia Zamudio-Haas, Breonna McCree, Azize Ngo, Akira Jackson, Carla Clynes, Luz Venegas, Arianna Salinas, Cinthya Herrera, et al. 2020. Research with marginalized communities: challenges to continuity during the COVID-19 pandemic. AIDS and Behavior 24, 7 (2020), 2009-2012. https://doi.org/10.1007/s10461-020-02920-3

[70] Kai Shu, Amy Sliva, Suhang Wang, Jiliang Tang, and Huan Liu. 2017. Fake news detection on social media: A data mining perspective. ACM SIGKDD explorations newsletter 19, 1 (2017), 22-36. https://doi.org/10.1145/3137597.3137600

[71] Simon, Sara. 2021. Inconsistent Reporting Practices Hampered Our Ability to Analyze COVID-19 Data. Here Are Three Common Problems We Identified. Retrieved 2021-09-06 from https://covidtracking.com/analysis-updates/threecovid-19-data-problems

[72] Alexander Skulmowski and Bernhard Standl. 2021. COVID-19 information fatigue? A case study of a German university website during two waves of the pandemic. Human behavior and emerging technologies 3, 3 (2021), 350-356.

[73] Robert M Sloyan. 2009. Trust, sensemaking, and individual responses to organizational change.

[74] Susan Leigh Star and Karen Ruhleder. 1996. Steps toward an ecology of infrastructure: Design and access for large information spaces. Information systems research 7, 1 (1996), 111-134.

[75] Kate Starbird, Jim Maddock, Mania Orand, Peg Achterman, and Robert M Mason. 2014. Rumors, false flags, and digital vigilantes: Misinformation on twitter after the 2013 boston marathon bombing. https://doi.org/10.9776/14308

[76] David Sterret, Dan Malato, Jennifer Benz, Liz Kantor, Trevor Tompson, Tom Rosenstiel, Jeff Sonderman, Kevin Loker, and Emily Swanson. 2018. Who shared it? How Americans decide what news to trust on social media. Technical Report. Norc Working Paper Series, WP-2018-001, 1-24.

[77] Nurul Suhaimi, Nutchanon Yongsatianchot, Yixuan Zhang, Anisa Amiji, Shivani A. Patel, Stacy Marsella, Miso Kim, Jacqueline Griffin, and Andrea Parker. 2020. Examining Older Adults' Information Exposure, Wellbeing, and Adherence to Protective Measures During the COVID-19 Pandemic. https: //arxiv.org/abs/2112.11215 Accessed: 2021-12-21.

[78] Samia Tasnim, Md Mahbub Hossain, and Hoimonty Mazumder. 2020. Impact of rumors and misinformation on COVID-19 in social media. Fournal of preventive medicine and public health 53, 3 (2020), 171-174. https://doi.org/10.3961/jpmph. 20.094

[79] The Cell Editorial Team. 2020. COVID-19: Navigating Uncertainties Together Cell 181, 2 (2020), 209. https://doi.org/10.1016/j.cell.2020.03.041

[80] The COVID Tracking Project. 2021. The COVID Tracking Project. https //covidtracking com Accessed: 2021-09-06.

[81] The New York Times. 2021. Coronavirus in the U.S.: Latest Map and Case Count Retrieved 2021-09-05 from https://www.nytimes.com/interactive/2021/us/covid cases.html

[82] The New York Times. 2021. Pulitzer Prizes Focus on Coverage of Pandemic and Law Enforceme. https://www.nytimes.com/2021/06/11/business/media/pulitzerprizes.html. Accessed: 2021-08-12.

[83] The Washington Post. 2021. How CDC data problems put the U.S. behind on the delta variant. Retrieved 2021-09-06 from https://www.washingtonpost. com/health/2021/08/18/cdc-data-delay-delta-variant

[84] The Washington Post. 2021. People are more anti-vaccine if they get their covid news from Facebook than from Fox News, data shows. Retrieved July 27, 2021 from https://www.washingtonpost.com/politics/2021/07/27/people-aremore-anti-vaccine-if-they-get-their-covid-19-news-facebook-rather-thanfox-news-new-data-shows

[85] David R Thomas. 2006. A general inductive approach for analyzing qualitative evaluation data. American journal of evaluation 27, 2 (2006), 237-246.

[86] Kimberley Thomas, R Dean Hardy, Heather Lazrus, Michael Mendez, Ben Orlove Isabel Rivera-Collazo, J Timmons Roberts, Marcy Rockman, Benjamin P Warner, and Robert Winthrop. 2019. Explaining differential vulnerability to climate change: A social science review. Wiley Interdisciplinary Reviews: Climate Change
10, 2 (2019), e565. https://doi.org/10.1002/wcc. 565

[87] University of California San Francisco. 2021. UCSF to House COVID Tracking Project, a National Database Donated by The Atlantic. Retrieved 2021-08-12 from https://www.ucsf.edu/news/2021/07/421151/ucsf-house-covid-trackingproject-national-database-donated-atlantic

[88] U.S. Department of Health \& Human Services. 2020. 2020 Percentage Poverty Tool. https://aspe.hhs.gov/system/files/aspe-files/107166/2020-percentagepoverty-tool.pdf

[89] U.S. Food and Nutrition Service. 2020. 85 Fed. Reg. 16,050. https://www.govinfo. gov/content/pkg/FR-2020-03-20/pdf/2020-05982.pdf

[90] Tavish Vaidya, Daniel Votipka, Michelle L. Mazurek, and Micah Sherr. 2019. Does Being Verified Make You More Credible? Account Verification's Effect on Tweet Credibility. Association for Computing Machinery, New York, NY, USA, 1-13. https://doi.org/10.1145/3290605.3300755

[91] Sander Van der Linden, Costas Panagopoulos, Flávio Azevedo, and John T Jost. 2021. The paranoid style in American politics revisited: An ideological asymmetry in conspiratorial thinking. Political Psychology 42, 1 (2021), 23-51. https://doi.org/10.1017/S0033291720001890

[92] Jan-Willem van Prooijen, Jaap Staman, and André PM Krouwel. 2018. Increased conspiracy beliefs among ethnic and Muslim minorities. Applied cognitive psychology 32, 5 (2018), 661-667. https://doi.org/10.1002/acp.3442

[93] Elaine Vaughan and Timothy Tinker. 2009. Effective health risk communication about pandemic influenza for vulnerable populations. American journal of public health 99, S2 (2009), S324-S332. https://doi.org/10.2105/AJPH.2009.162537

[94] Tiffany C Veinot, Hannah Mitchell, and Jessica S Ancker. 2018. Good intentions are not enough: how informatics interventions can worsen inequality. Fournal of the American Medical Informatics Association 25, 8 (2018), 1080-1088. https: //doi.org/10.1093/jamia/ocy052

[95] Sam Wineburg and Sarah McGrew. 2016. Evaluating information: The cornerstone of civic online reasoning.

[96] Frankie Ho Chun Wong, Tianyin Liu, Dara Kiu Yi Leung, Anna Y Zhang, Walker Siu Hong Au, Wai Wai Kwok, Angie KY Shum, Gloria Hoi Yan Wong, and Terry Yat-Sang Lum. 2021. Consuming information related to COVID-19 on social media among older adults and its association with anxiety, social trust in information, and COVID-safe behaviors: Cross-sectional telephone survey. fournal of Medical Internet Research 23, 2 (2021), e26570. https://doi.org/10.2196/ 26570

[97] Liang Wu, Fred Morstatter, Kathleen M Carley, and Huan Liu. 2019. Misinformation in social media: definition, manipulation, and detection. ACM SIGKDD Explorations Newsletter 21, 2 (2019), 80-90. https://doi.org/10.1145/3373464. 3373475

[98] Yi Wu and Fei Shen. 2021. Exploring the impacts of media use and media trust on health behaviors during the COVID-19 pandemic in China. Journal of health psychology 0, 0 (2021), $1359105321995964-1359105321995964$.

[99] Waheeb Yaqub, Otari Kakhidze, Morgan L. Brockman, Nasir Memon, and Sameer Patil. 2020. Effects of Credibility Indicators on Social Media News Sharing Intent. In Proceedings of the 2020 CHI Conference on Human Factors in Computing Systems (Honolulu, HI, USA) (CHI '20). Association for Computing Machinery, New York, NY, USA, 1-14. https://doi.org/10.1145/3313831.3376213

[100] Yixuan Zhang, Nurul Suhaimi, Rana Azghandi, Mary Amulya Joseph, Miso Kim, Jacqueline Griffin, and Andrea G. Parker. 2020. Understanding the Use of Crisis Informatics Technology among Older Adults. In Proceedings of the 2020 CHI Conference on Human Factors in Computing Systems (Honolulu, HI, USA) (CHI '20). Association for Computing Machinery, New York, NY, USA, 1-13. https://doi.org/10.1145/3313831.3376862

[101] Yixuan Zhang, Yifan Sun, Lace Padilla, Sumit Barua, Enrico Bertini, and Andrea G Parker. 2021. Mapping the Landscape of COVID-19 Crisis Visualizations. In Proceedings of the 2021 CHI Conference on Human Factors in Computing Systems (Yokohama, Japan) (CHI '21). Association for Computing Machinery, New York, NY, USA, Article 608, 23 pages. https://doi.org/10.1145/3411764.3445381 